\title{
A neurodegenerative disease landscape of rare mutations in Colombia due to founder effects
}

\author{
Juliana Acosta-Uribe ${ }^{1,2 \dagger}$, David Aguillón²†, J. Nicholas Cochran³ ${ }^{3}$ Margarita Giraldo², , Lucía Madrigal², \\ Bradley W. Killingsworth', Rijul Singhal ${ }^{1}$, Sarah Labib ', Diana Alzate², Lina Velilla², Sonia Moreno², \\ Gloria P. García², Amanda Saldarriaga², Francisco Piedrahita², Liliana Hincapié2, Hugo E. López², \\ Nithesh Perumal', Leonilde Morelo ${ }^{5}$, Dionis Vallejo ${ }^{6}$, Juan Marcos Solano ${ }^{6}$, Eric M. Reiman${ }^{7}$, Ezequiel I. Surace ${ }^{8}$, \\ Tatiana Itzcovich ${ }^{8}$, Ricardo Allegri ${ }^{9}$, Raquel Sánchez-Valle ${ }^{10}$, Andrés Villegas-Lanau ${ }^{2}$, Charles L. White III ${ }^{11}$, \\ Diana Matallana ${ }^{12,13}$, Richard M. Myers ${ }^{3}$, Sharon R. Browning ${ }^{14}$, Francisco Lopera ${ }^{2^{* \dagger}}$ and Kenneth S. Kosik ${ }^{1 * \dagger}$
}

\begin{abstract}
Background: The Colombian population, as well as those in other Latin American regions, arose from a recent tricontinental admixture among Native Americans, Spanish invaders, and enslaved Africans, all of whom passed through a population bottleneck due to widespread infectious diseases that left small isolated local settlements. As a result, the current population reflects multiple founder effects derived from diverse ancestries.

Methods: We characterized the role of admixture and founder effects on the origination of the mutational landscape that led to neurodegenerative disorders under these historical circumstances. Genomes from 900 Colombian individuals with Alzheimer's disease (AD) $[n=376]$, frontotemporal lobar degeneration-motor neuron disease continuum (FTLD-MND) [ $n=197]$, early-onset dementia not otherwise specified (EOD) $[n=73]$, and healthy participants $[n=$ 254] were analyzed. We examined their global and local ancestry proportions and screened this cohort for deleterious variants in disease-causing and risk-conferring genes.

Results: We identified 21 pathogenic variants in AD-FTLD related genes, and PSEN1 harbored the majority (11 pathogenic variants). Variants were identified from all three continental ancestries. TREM2 heterozygous and homozygous variants were the most common among AD risk genes (102 carriers), a point of interest because the disease risk conferred by these variants differed according to ancestry. Several gene variants that have a known association with MND in European populations had FTLD phenotypes on a Native American haplotype. Consistent with founder effects, identity by descent among carriers of the same variant was frequent.
\end{abstract}

\footnotetext{
*Correspondence: francisco.lopera@gna.org.co; kosik@ucsb.edu

†Juliana Acosta-Uribe, David Aguillón, Francisco Lopera and Kenneth S.

Kosik contributed equally to this work.

${ }^{1}$ Neuroscience Research Institute and Department of Molecular Cellular and Developmental Biology, University of California, Santa Barbara, CA, USA

${ }^{2}$ Grupo de Neurociencias de Antioquia, School of Medicine, Universidad

de Antioquia, Medellín, Colombia

Full list of author information is available at the end of the article
} original author(s) and the source, provide a link to the Creative Commons licence, and indicate if changes were made. The images or other third party material in this article are included in the article's Creative Commons licence, unless indicated otherwise in a credit line to the material. If material is not included in the article's Creative Commons licence and your intended use is not permitted by statutory regulation or exceeds the permitted use, you will need to obtain permission directly from the copyright holder. To view a copy of this licence, visit http://creativecommons.org/licenses/by/4.0/. The Creative Commons Public Domain Dedication waiver (http://creativeco mmons.org/publicdomain/zero/1.0/) applies to the data made available in this article, unless otherwise stated in a credit line to the data. 
Conclusions: Colombian demography with multiple mini-bottlenecks probably enhanced the detection of founder events and left a proportionally higher frequency of rare variants derived from the ancestral populations. These findings demonstrate the role of genomically defined ancestry in phenotypic disease expression, a phenotypic range of different rare mutations in the same gene, and further emphasize the importance of inclusiveness in genetic studies.

Keywords: Founder effect, Bottleneck, Admixture, Genetic drift, Selection, Demography, Neurodegeneration, Alzheimer's disease, Frontotemporal dementia, Motor neuron disease

\section{Background}

The circumstances related to Latin America's unique demographic history led to numerous genetic founders that expanded rare genetic variation. The regional populations of Colombia originated from varying proportions of a recent tri-continental admixture consisting of diverse indigenous peoples, Spanish invaders, and enslaved Africans, all of whom had been geographically separated for tens of thousands of years. During the Spanish conquest, these individuals suffered massive mortality from numerous infectious diseases, including smallpox, influenza, syphilis, hepatitis, measles, encephalitis, tuberculosis, diphtheria, cholera, typhus, scarlet fever, and meningitis, which created a narrow bottleneck with a minimum effective population size approximately 12 generations ago [1]. Survivors were geographically dispersed in a patchwork of relatively isolated small founder populations. Following the first decades of the Spanish invasion and European expansion throughout various territories, the second half of the sixteenth century saw a large and continuous growth of an admixed population, especially in the Andean region of the country (Additional file 1: Figure S1). The population growth amplified the effects of genetic drift confined to highly local settings that marked a fine-grained geographic map with a local genetic stamp [2].

Demographic history and local ancestry have gained significant interest in genomic studies aiming to understand the disease burden of underrepresented populations and transferability of risk scores from research done in European cohorts. However, most of these studies have focused on genome wide association studies (GWAS) and polygenic risk scores that usually rely on the sequencing of common genetic variants [3-5], while missing those rare alleles absent from European genomes [6]. Rare variants are likely to play a role in the problem of "missing heritability," have larger effect sizes [7], and are more susceptible to population dynamics and genetic drift.

Rare mutations contribute to the occurrence of neurodegenerative disease, which prompted a search for individuals with young onset familial dementia and related neurodegenerative disorders. We suspected that genetic drift stamped local populations with unique sets of rare variants. Numerous rare genetic conditions converge under this phenotypic label, and therefore as a population indicator of rare variation, dementia represents a readily identifiable trait with a great deal of genetic variation. Among the many genes in which disease mutations fit the phenotypic label are PSEN1 [MIM: 104311], PSEN2 [MIM: 600759], APP [MIM: 104760], C9orf72 [MIM: 614260], GRN [MIM: 138945], MAPT [MIM: 157140], TARDBP [MIM: 605078], FUS [MIM: 137070], VCP [MIM: 601023], CHMP2B [MIM: 609512], and TBK1 [MIM: 604834] [8]. Rare variants in these genes offer novel perspectives on the breadth of their associated clinical phenotypes and the underlying molecular pathways. Here, we describe a cohort of 900 Colombian individuals with neurodegenerative diseases and report the genetic variants associated with neurodegeneration in the context of their ancestral origins and admixture.

\section{Methods \\ Subjects}

Participants were recruited or referred to the "Grupo de Neurociencias de Antioquia," University of Antioquia, Colombia for "The Admixture and Neurodegeneration Genomic Landscape" (TANGL) study. The project was approved by the Institutional Review Board (IRB) of the Medical Research institute, School of Medicine, Universidad de Antioquia. Written informed consent following the guidelines of the Code of Ethics of the World Medical Association, Helsinki declaration, and Belmont Report was obtained from all participants or their legally authorized proxies. The recruitment targeted patients with early-onset dementia and families in which multiple first-degree relatives were affected. All the individuals were born in Colombia (Additional file 1: Figure S1). All subjects were evaluated following a standard protocol including physical and neurological examination, as well as population validated neuropsychological assessment $[9,10]$. Family history was obtained from the patients and their relatives and was considered positive if at least one first or second degree relative presented dementia or motor neuron disease (MND). Families were classified as autosomal dominant if at least three first degree relatives suffered from dementia or MND in two consecutive generations. When patients had familial forms of dementia, 
their relatives with neurological and psychiatric disorders were recruited along with healthy family members. Nine hundred individuals from 566 families with high quality genomes were used for analyses (genetic sequencing and quality control procedures are detailed in the Genome Sequencing methods).

Based on their clinical diagnosis, participants were divided in four cohorts:

- The Alzheimer's disease (AD) [MIM: 104300] cohort ( $n=376)$ included individuals with early-onset $\mathrm{AD}$ (AAO $\leq 65$ years) and individuals with autosomal dominant late onset $\mathrm{AD}$. Patients with atypical presentations of $\mathrm{AD}$, such as primary progressive aphasia-logopenic variant (lvPPA), posterior cortical atrophy, and spastic paraparesis associated with PSEN1 pathogenic variants [MIM: 607822] were included in this cohort. AD was diagnosed according the NINCDS-ADRDA criteria [11].

- The frontotemporal lobar degeneration and motor neuron disease (FTLD-MND) spectrum cohort ( $n$ $=197$ ) comprised patients with multiple presentations of frontotemporal lobar degeneration (FTLD) [MIM: 600274], which include behavioral variant of frontotemporal dementia (bvFTD), primary progressive aphasia-semantic variant (svPPA), primary progressive aphasia-non-fluent/agrammatic variant (navPPA), and FTLD with amyotrophic lateral sclerosis (FTLD-ALS). Diagnosis of FTLD variants was done according to Gorno-Tempini et al. 2011 [12] and Rascovsky et al. 2011 [13]. Patients with corticobasal degeneration (CBD), progressive supranuclear palsy (PSP) [MIM: 601104] diagnosed according to The Movement Disorder Society Criteria [14], and with amyotrophic lateral sclerosis (ALS) [MIM: 105400], diagnosed according to Strong et al. 2017 [15], were included in this cohort.
- The early-onset dementia not otherwise specified (EOD) cohort $(n=73)$ included patients with earlyonset dementia (AAO $\leq 65$ years) that did not fully meet criteria for AD or FTLD at the time of evaluation and did not have secondary causes that explain their neurodegeneration. Some of these individuals were relatives of the patients from the other cohorts but presented with conditions such as Parkinson's disease [MIM: 168600], bipolar disorder [MIM: 125480], or Lewy body disease [MIM: 127750].

- The Healthy participant cohort $(n=254)$ included individuals related and unrelated to the patients. These subjects had a Clinical Dementia Rating (CDR) score of 0 in their last examination and no evidence of neurodegenerative dementia or motor neuron disease.

The complete demographic information of the 900 individuals can be found in Table 1, Additional file 2: Table S1 and Additional file 3: Table S2.

\section{Genome sequencing}

Peripheral blood from the participants was obtained by standard phlebotomy, and genomic DNA was isolated from leukocytes using the Gentra Puregene Blood Kit (Qiagen). Genome sequencing (WGS) was performed at the HudsonAlpha Institute for Biotechnology on either the Illumina HiSeq X platform, or the Illumina NovaSeq platform. A subset of individuals was sequenced at the Human Longevity Institute on the Illumina HiSeq X platform (119 samples). The combined dataset had a mean read depth of $34 \mathrm{X}$ and an average of $92 \%$ of bases covered at 20X. Sequencing libraries at HudsonAlpha were prepared by Covaris shearing, end repair, adapter ligation, and PCR using standard protocols. Library concentrations were normalized using KAPA qPCR prior to sequencing. Sequencing reads from both centers were

Table 1 Demographic information of the included cohorts

\begin{tabular}{|c|c|c|c|c|c|c|c|c|c|c|c|c|c|c|c|c|c|}
\hline \multirow[t]{3}{*}{ Cohort } & \multirow[t]{3}{*}{$n$} & \multirow{2}{*}{\multicolumn{2}{|c|}{ AAO }} & \multirow{2}{*}{\multicolumn{2}{|c|}{ Female }} & \multicolumn{12}{|c|}{ APOE genotype no. (\%) } \\
\hline & & & & & & \multicolumn{2}{|c|}{$\epsilon 2 / \epsilon 2$} & \multicolumn{2}{|c|}{$\epsilon 2 / \epsilon 3$} & \multicolumn{2}{|c|}{$\epsilon 2 / \epsilon 4$} & \multicolumn{2}{|c|}{$\epsilon 3 / \epsilon 3$} & \multicolumn{2}{|c|}{$\epsilon 3 / \epsilon 4$} & \multicolumn{2}{|c|}{$\epsilon 4 / \epsilon 4$} \\
\hline & & Mean & Range & $n$ & $\%$ & $n$ & $\%$ & $n$ & $\%$ & $n$ & $\%$ & $n$ & $\%$ & $n$ & $\%$ & $n$ & $\%$ \\
\hline$A D$ & 376 & 59 & $30-90$ & 249 & 66.2 & - & - & 15 & 4 & 4 & 1.1 & 168 & 45 & 139 & 37.1 & 49 & 13.1 \\
\hline FTLD-MND & 197 & 58.8 & $21-82$ & 92 & 46.7 & 1 & 0.5 & 18 & 9.1 & - & - & 122 & 62 & 49 & 24.9 & 7 & 3.6 \\
\hline EOD & 73 & 54.5 & $25-75$ & 49 & 67.1 & - & - & 2 & 2.7 & - & - & 39 & 53 & 20 & 27.4 & 12 & 16.4 \\
\hline \multirow[t]{2}{*}{ Healthy } & 254 & 60 & $18-100^{a}$ & 159 & 62.6 & 2 & 0.8 & 25 & 9.8 & 1 & 0.4 & 159 & 45 & 61 & 23.9 & 4 & 1.6 \\
\hline & & & & 549 & 60.7 & 3 & 0.3 & 60 & 6.7 & 5 & 0.6 & 488 & 54.2 & 269 & 29.9 & 72 & 8 \\
\hline
\end{tabular}

$A D$ Alzheimer's disease, FTLD-MND frontotemporal lobar degeneration and motor neuron disorder, EOD early-onset dementia not otherwise specified, $A A O$ age at onset

a Age at evaluation. There were three Individuals with uncalled APOE genotype (one from AD cohort and two healthy individuals) 
aligned to the hg19 reference genome with bwa-0.7.12 [16]. BAMs were sorted and duplicates were marked with Sambamba 0.5.4 [17]. Indels were realigned, bases were recalibrated, and gVCFs were generated with GATK 3.3 [18]. Variants were called across all samples in a single batch with GATK 3.8 using the -newQual flag to minimize false negative singleton calls. The recall rate for GATK against truth sets is between 93 and $99 \%$ for single nucleotide variants and 85 and $98 \%$ for small (less than $50 \mathrm{bp}$ ) indel events [19]. Genome annotation was performed using SnpEff 4.3 [20] after splitting multi-allelic sites with $\mathrm{Vt}$ [21]. The genome was annotated with the gene definitions from human genome build Ensembl GRCh37.75 [22]. All single nucleotide variants and indels were annotated with CADD v1.3 [23]. Population database frequency annotations included 1000 Genomes Phase 3 (1000GP) [24], TOPMed Bravo [25] (lifted over from hg38 to hg19 using CrossMap 0.2.7 [26]), and several population database sets annotated using WGSA 0.7 [27] including ExAC [28], gnomAD [29], ESP [30], and UK10K [31]. Variants were also annotated with dbSNP release 151 [32].

Calls were filtered with vcftools (v0.1.12b) [33] to retain sites with quality scores equal or greater than 20 and mean read depth scores equal or greater than 30. KING (v2.2.4) [34] was used to verify disclosed familiar relationships and pedigree structures, and individuals with unexplained relatedness were removed. For duplicate samples and monozygotic twin pairs, only one genome was kept. PLINK v.1.90 [35, 36] was used to identify and exclude individuals with discordant X-chromosome sex and those with more than 5\% missing data [37]. Mendel errors were set to missing before removing autosomal variants with missingness $>5 \%$ obtaining a total of $41,123,431$ variants and 900 individuals from 566 families available for analysis (Additional file 1: Figure S2).

To compare the TANGL genomes to previously identified carriers of PSEN1 c.428T $>C$ (p.Ile143Thr) [38] from Colombia and PSEN1 c.356C $>\mathrm{T}$ (p.Thr119Ile) from Colombia and Argentina [39], we sequenced additional individuals using the Array-8+ v1.0 Kit + neuro booster array consortium (NBA) content, beadchip 20042459 Illumina Global Diversity (Catalog 20031816). Imputation was performed using the TOPMed Imputation Panel and Server (version 1.3.3) [40], which includes 97,256 references samples and 308,107,085 variants and uses Minimac4 for imputation. Pre-imputation scripts (version 4.3.0 from William Rayner at the University of Oxford) were run using default settings, which filtered out palindromic single nucleotide variants (SNVs) with minor allele frequency (MAF) $>0.4$ or variants with $>0.2$ MAF difference from the TOPMed reference panel [41]. The Colombian carriers of these PSEN1 variants had been recruited and evaluated by the Grupo de Neurociencias de Antioquia (GNA). The Argentinian sample was provided by the Neurodegenerative illnesses' laboratory (Fleni-CONICET). The clinical assessment and sequencing of these individuals was done with written informed consent and approved by the IRB of the Medical Research Institute School of Medicine, Universidad de Antioquia, and the IRB from "Instituto de Investigaciones Neurológicas Raúl Carrea - FLENI."

To compare the TANGL genomes to previously identified carriers of MAPT c.1189C $>\mathrm{T}$ (p.Pro397Ser) from Spain, we obtained exome sequencing data from an individual previously sequenced by the Alzheimer's disease and other cognitive disorders unit at Hospital Clínic de Barcelona. The exome from the Spanish c.1189C $>$ T (p.Pro397Ser) carrier [42] was processed from fastq to VCF using a standard clinical alignment pipeline from the HudsonAlpha Institute for Biotechnology Clinical Services Laboratory that uses Sentieon version 201808.07 (a computational wrapper for common tools such as bwa), including alignment with Sentieon-BWA (version 201808.07; identical to bwa mem 0.7.15-r1140) and variant calling with Illumina Strelka2 (version 2.9.10) [43]. The use of this sample was approved by the IRB from the "Hospital Clinic de Barcelona."

\section{Population structure analysis}

We implemented protocols similar to those previously developed for ancestry estimation in admixed populations [3, 44]. We merged the 900 genomes (TANGL cohort) with the 1000 Genomes Project (1000GP) Phase 3 genomes generating the TANGL.1000GP dataset ( $n$ $=3404)$. Then, we created a subset including only the TANGL cohort, the non-admixed African Populations (AFR), $N=504$, and European populations (EUR), $N=$ 503. We merged these genomes with Native American samples (NAT), $N=43$ from Mao et al. [45] inferred to have $>0.99$ Native Ancestry, and created the TANGL. AFR.EUR.NAT dataset. After removing monomorphic variants, triallelic sites that were not due to a strand flip in either dataset and those sites with missingness greater than or equal to $1 \%$, we retained 845,950 autosomal variants and 1950 individuals for further analysis.

\section{Global ancestry inference}

A subset of unrelated samples from TANGL.AFR.EUR. NAT was selected by keeping only the proband of each family and, using KING (v2.2.4) [34] with "-related" and “--degree 3" settings to identify cryptic relatedness. Only sample pairs with kinship coefficient less than 0.044 were retained for TANGL, AFR and EUR. The NAT individuals showed significant relatedness between them, and the threshold for that population was set to "-degree 2" 
to retain the most NAT samples with kinship less than 0.0884. The final TANGL.AFR.EUR.NAT -Unrelated dataset comprised 1611 unrelated individuals (TANGL $N$ $=566, \operatorname{AFR} N=501$, EUR $N=503$, NAT $=41$ ).

We calculated global ancestry using ADMIXTURE (v.1.3.0) [46] independently for the unrelated TANGL individuals $(n=566)$ and for the TANGL.AFR.EUR. NAT-Unrelated cohort. As recommended by ADMIXTURE, PLINK (v.1.9) $[35,36]$ was used to perform pairphased linkage disequilibrium (LD) pruning; excluding variants with an $r^{2}$ value of greater than 0.2 with any other SNP within a 50-SNP sliding window, advancing by 10 SNPs each time (--indep-pairwise 5010 0.2). The LDpruned dataset contained 203,810 variants. We then performed an unsupervised analysis modeling from one to ten ancestral populations $(K=1-10)$ using the random seed option and replicating each calculation 20 times. We selected the run with the best Loglikehood value for each $K$ and compared the cross validation (cv) error values to determine the model with the lowest cv value. Ancestral proportion statistics of mean and standard deviation were calculated using the statistical software R [47].

In addition, we determined mitochondrial and Y-chromosome haplogroups of the TANGL-unrelated cohort using HaploGrep 2 with Phylotree 17 [48], and yHaplo respectively [49].

\section{Local ancestry inference}

We phased the combined TANGL.AFR.EUR.NAT dataset with SHAPEIT (v.2.r900) [50] using the haplotype reference panel of the 1000GP. We used the parameters -duohmm and a window of $5 \mathrm{MB}(-\mathrm{W} 5)$, which takes advantage of the inclusion of families, pedigree structure, and the large amount of IBD shared by close relatives, leading to increased accuracy [51]. We used the PopPhased version of RFMix (v1.5.4) [52] to estimate the local ancestry using the following flags: $-\mathrm{w} 0.2$, -e 1 , -n 5, --use-reference-panels-in-EM, --forward-backward as recommended by Martin et al. [3] for estimating local ancestry in admixed populations. To determine the carrier haplotype and local ancestry of a rare variant of interest, we used PLINK (v.1.9) [35, 36]. We identified other single nucleotide variants (SNVs) in linkage disequilibrium with the variant of interest and used them as tags to identify the carrier haplotypes in the phased dataset, and then searched for the local ancestry of the specific locus in the RFMix output.

\section{Principal component analysis (PCA)}

For PCA, we used the subset of unrelated samples with LD-pruning of variants as described in the methods for "Global ancestry inference." We performed a PCA using the smartpca package from EIGENSOFT (v7.2.1) [53], with 3 outlier removal iterations (numoutlieriter: 3 ) and flag "altnormstyle: NO" to match EIGENSTRAT normalization formulas [53]. The PCA results were plotted using the PCAviz package [54] for R. For the PCA with the Ancestral populations, we retained variants with MAF $>10 \%$. For the PCA of the TANGL-unrelated cohort, we extracted a common variant set, retaining those with MAF $>10 \%$, and then a lower frequency variant set, keeping only variants with MAF between 5 and $10 \%$.

\section{Genetic screening for disease causing variants}

Each individual was initially screened for pathogenic variants in the most recognized genes associated with $\mathrm{AD}$ and FTLD according to AD/FTLD mutation databases (https://www.molgen.vib-ua.be/ADMutations, https:// www.alzforum.org/mutations); PSEN1, PSEN2, APP, $M A P T, G R N$ VCP, FUS, CHMP2B, TARDBP, and TBK1 (the molgen.vib-ua.be/ADMutations database is not available as of July 2021). For the present study, the terms "pathogenic" and "likely pathogenic" refer to variants that are both predicted to be disruptive or damaging to the protein function and causative for a disease according ACMG criteria [55].

A secondary genetic analysis was done to identify pathogenic and likely pathogenic variants in other genes associated with similar or overlapping phenotypes. For the secondary screening, we chose the disease-causing genes reported in the following OMIM phenotypic series and phenotypes: frontotemporal dementia and/or amyotrophic lateral sclerosis [MIM: PS105550, PS167320, PS105400], Parkinson disease [MIM: PS168600], adultonset leukoencephalopathies [MIM: PS125310, 221820], and ceroid lipofuscinoses [MIM: PS256730]. We retained variants with MAF of 0.001 or less in the ExAC database if the gene had autosomal dominant or X-linked inheritance, and 0.01 or less if the gene had autosomal recessive inheritance. The remaining variants were discarded if they were more prevalent in controls than cases or if they had a CADD Phred score less than 20. The selected protein altering variants defined as nonsynonymous single nucleotide variants, splicing altering variants, insertions, or deletions were manually curated by searching in the databases described before as well as ClinVar [56] and LitVar [57]. The previously unreported (novel) variants were classified according to the guidelines published by the American College of Medical Genetics and Genomics and the Association for Molecular Pathology [55]. Variants in PSEN1 and PSEN2 were also classified according the Guerreiro algorithm [58]. Additionally, subjects were screened for C9ORF72 [MIM: 614260] hexanucleotide expansion using repeat-primer following the protocol described in DeJesus-Hernandez et al. [59] because, while C9ORF72 expansions are possible to detect from 
short-read PCR-free genomes [60], such events are not detectable from PCR positive genomes which were conducted here. We searched for large copy number variations using four callers: DELLY [61], ERDS [62], CNVnator [63], and BIC-seq2 [64]. Events called by multiple callers were inspected for validity using Integrative Genomics Viewer [65]. In contrast to GATK small variant calls, where recall rates against truth sets are known, there are not recall rates available for this employed combination of tools, though we note that there is a high false negative rate for all CNV callers from short read PCRpositive genome data; thus, the goal in $\mathrm{CNV}$ analysis was to have high confidence in those variants that were identifiable across all four callers at the expense of missing some true positives that may not pass these strict criteria. Better detection of expansions such as C9ORF72 or heretofore unidentified similar events and/or better large indel detection will be aided by emerging use of long read sequencing which can help identify events that would be missed otherwise [66].

Neuropathologic assessment of CSF1R c.2068G>A (p.Gly690Ser) and DNAJC5 c.347 T>G (p.Leu116Arg) carriers was performed at the Brain Bank of the Neuroscience Group of Antioquia following standardized protocols $[67,68]$. Tissues were stained with hematoxylin-eosin, Luxol Fast blue, and periodic acid-Shiff (PAS). The brain donation and neuropathologic assessment were done with written informed consent and approved by the IRB of the Medical Research Institute School of Medicine, Universidad de Antioquia.

\section{Genetic screening for risk associated variants}

We used publications in the literature to identify genes in which rare variants were associated with increased risk for $\mathrm{AD}$ and/or FTLD-MND with an odds ratio higher than 2. TREM2 [69, 70] [MIM: 605086], ABCA7 [69, 71, 72] [MIM: 107741], and SORL1 [69, 73] [MIM: 602005] were selected as intermediate effect risk genes. We retained variants that were known to be risk conferring, led to premature truncation of the protein (PTV), or that were classified as strictly damaging (SD) according to previous published criteria [69]. Strictly damaging variants had MAF $\leq 0.01$ in the ExAC database and were unanimously classified as deleterious by three different in silico prediction algorithms; SIFT [74], Polyphen-2 (Hum Div.) [75], and MutationTaster [76] In addition to this strategy, we included $A D A M 10$ [MIM: 602192 ] c.510G $>$ T (p.Gln170His) and c.541A $>\mathrm{T}$ (p.Arg181Gly) variants as they have been reported to confer intermediate risk for AD [77, 78]. Variant nomenclature is according to the Human Genome Variation Society Recommendations [79]; the GenBank reference transcripts used for each disease causing and risk conferring variant can be found in Additional file 4: Table S3.

\section{Identity by descent}

If any of the disease-conferring or risk-associated variants were shared by two or more unrelated individuals, we used hap-IBD [80] v1.0 to search for identity by descent (IBD) around the locus. Because this software detects IBD of $2 \mathrm{cM}$ and higher, we additionally performed an alignment of the haplotypes carrying the variants of interest to search for smaller IBD segments between the TANGL and 1000 Genomes Project (1000GP) carriers. Autozygosity (homozygosity by descent) was determined using the same methods. Code and scripts used for the population structure and identity by descent analyses are publicly available [81].

\section{Results}

\section{Population analysis of the genomes}

\section{from the neurodegeneration cohort}

Nine hundred Colombian individuals with high-quality genome sequences were included in "The Admixture and Neurodegeneration Genomic Landscape" (TANGL) study. The individuals were divided into four different cohorts: Alzheimer's disease (AD), frontotemporal lobar degeneration and motor neuron disease (FTLD-MND), early-onset dementia not otherwise specified (EOD), and healthy participants (Table 1 and Additional file 2: Table S1). These 900 individuals represented 566 independent families, which were classified into the same four cohorts according to the diagnosis of the proband (Additional file 3: Table S2).

Because the sample set was highly selected, we first sought to determine the genomic similarity between the TANGL cohort and other Colombian individuals. We initially merged the TANGL and the 1000 Genomes Project (1000GP) phase 3 [82] datasets and performed a principal component analysis (PCA). The TANGL cohort had a similar distribution in the first three principal components (PC) to the "Colombians from Medellín" (CLM) of the 1000GP, allowing us to conclude that both populations are genetically similar (Additional file 1: Figure S3). To take a closer look into the ancestral origins of the TANGL cohort, we used the software ADMIXTURE to estimate the number of ancestral populations $(K)$ from which the cohort arose. The lowest cross validation (cv) error was obtained when assuming the cohort was derived from three ancestral populations $(k=3)$, which agrees with the history of the tri-continental admixture after the Spanish conquest (Additional file 1: Figure S4). To analyze the global and local ancestry of the TANGL cohort, we merged the TANGL genomes with the European and African populations from the 1000GP 
and Native American genomes from Mao et al. [45] and repeated the ADMIXTURE analysis. In this joint dataset, $K=3$ accurately differentiated Native American, European and African cohorts, but the lowest $\mathrm{CV}$ error was obtained for $K=6$ (Fig. 1 and Additional file 1: Figure S5). Modeling for six ancestral populations allowed the detection of substructure within the African and European cohorts and created an additional cluster described by Moreno-Estrada et al. [44] as a "Latino-specific European component." Consistent with previous studies [83], the ancestral population with the highest proportion in our cohort was European (mean of $64 \%, \mathrm{SD}=15 \%$ ), followed by Native American (mean of $27 \%, \mathrm{SD}=11 \%$ ), and African being the least represented (mean of 9\%, SD $=11 \%$ ) (Additional file 1: Figure S6). These individual admixture values ( $Q$-values) at $K=3$ correlated with the sum of local ancestries estimated by RFMix (Pearson's $r>0.99$ ), allowing us to conclude that the local ancestry inferred for each individual matches the percentages of global ancestry obtained by an orthogonal method) (Additional file 1: Figure S7). However, the regional differences in the fine structure of the Colombian population make these global ancestry proportions highly region dependent. For example, the three individuals whose global ancestry was nearly $90 \%$ African were from the Pacific coast of the country where former enslaved Africans settled and most of the population self identifies as Afro-Colombian (Additional file 1: Figure S1).

After calculating the proportions of global ancestry, we evaluated the TANGL cohort for sex biased admixture,
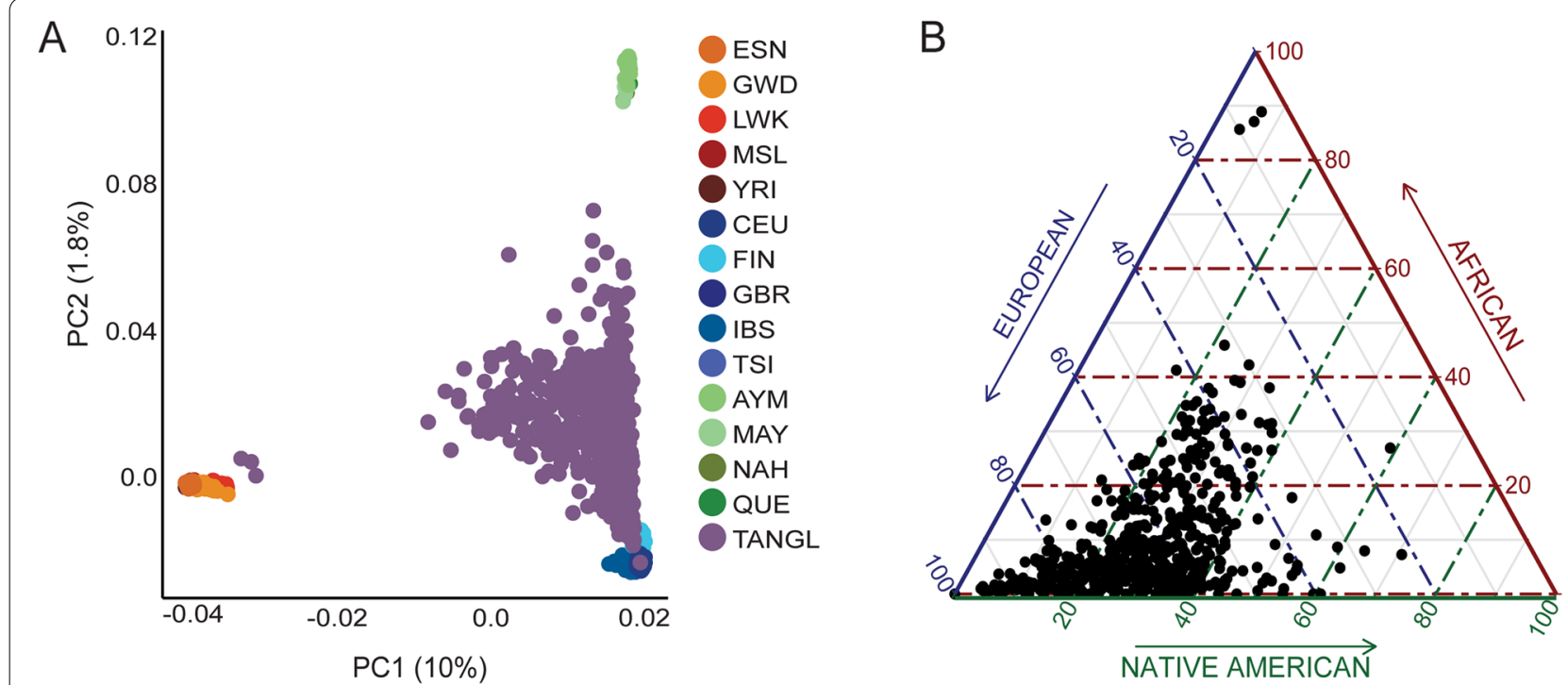

C

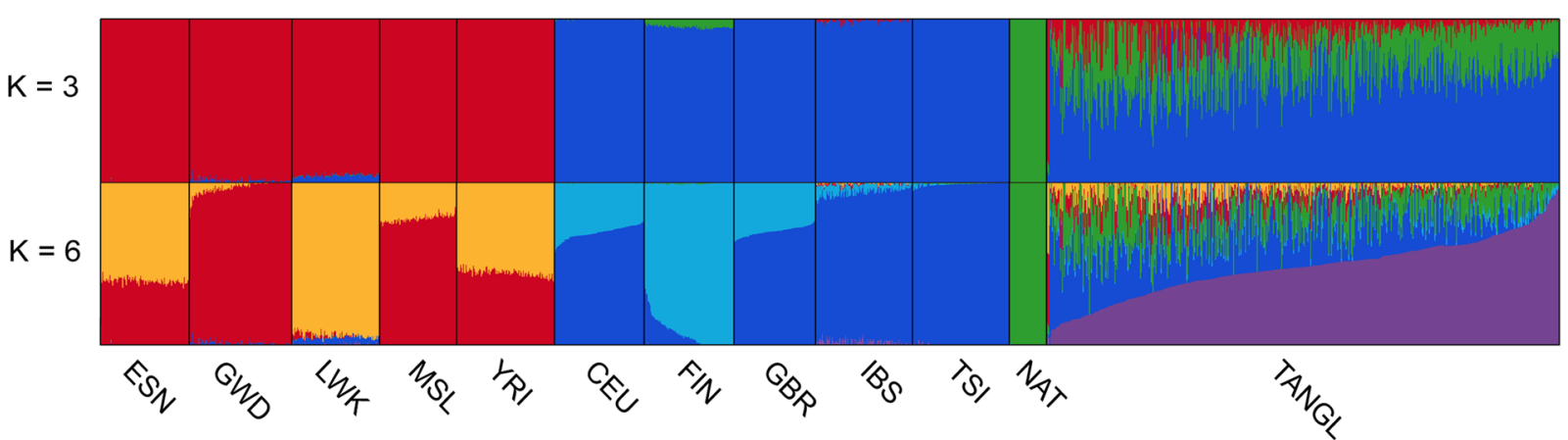

Fig. 1 Population structure and admixture analyses of the TANGL cohort. A PC1 vs PC2 of the PCA of the TANGL cohort (purple) with the European (blue) and African (orange) individuals from the 1000GP and 43 Native American genomes (green). B Ternary plot representing the global ancestry of each of the individuals in the TANGL cohort values according to sum of local ancestries calculated by RFMix. C Q plot of ADMIXTURE results assuming 3 and 6 ancestral populations (K). ESN: Esan in Nigeria. GWD: Gambian in Western Divisions in the Gambia. LWK: Luhya in Webuye, Kenya. MSL: Mende in Sierra Leone. YRI: Yoruba in Ibadan, Nigeria. CEU: Utah Residents (CEPH) with Northern and Western European Ancestry. FIN: Finnish in Finland. GBR: British in England and Scotland. IBS: Iberian Population in Spain. TSI: Toscani in Italia. AYM: Aymara. MAY: Mayan, NAH: Nahuatl. QUE: Quechua. NAT: Native American 
a genetic trait previously described in the Colombian population [84, 85]. We used HaploGrep2 and yHaplo to determine mitochondrial and Y-chromosome haplogroups. The mitochondrial haplogroups of the probands $(n=566)$ were predominantly Native American (83.4\%) while the Y-chromosome haplogroups $(n=224)$ were mostly of European and of Mediterranean origins (92.8\%), thus supporting the conclusion than multiple cohorts of Colombian origin show sex-biased admixture with Native American maternal lineages and paternal lineages from Europe (Additional file 5: Table S4 and Additional file 6: Table S5). Overall, these analyses let us conclude that despite recruiting the TANGL cohort based upon neurodegenerative conditions from the Andes region of Colombia, it recapitulated the admixture patterns previously described in the country.

The TANGL cohort was distributed between the three ancestral populations in the PCA, clustering closer to Europeans and Native Americans. To determine if the clustering of the admixed individuals was driven by their percentages of global ancestry, we compared the values of the principal components $(\mathrm{PC})$ with the percentage of global ancestry attributed to each of the three ancestral populations by ADMIXTURE. PC1 correlated with the percentage of African ancestry (Pearson's r2 of 1), and $\mathrm{PC} 2$ showed a correlation with the level of Native American ancestry (Pearson's r2 of 0.87) (Additional file 1: Figures S8, S9 and S10). To determine whether the Colombian population clustered according to their global ancestry without including the ancestral populations in the analyses, we retained the 566 unrelated probands from the TANGL cohort and performed two PCAs, one with common variants (MAF $>10 \%$ ) and one with less frequent variants (MAF 5-10\%). Both PCAs showed correlation of the PCs with the global admixture proportions, regardless of the inclusion of the ancestral population (Additional file 1: Figures S11, S12 and S13).

\section{Neurodegenerative disease variants in the TANGL cohort $A D$-associated genes}

The 900 genomes were initially examined for variants in AD-associated genes (PSEN1, PSEN2, and APP), and the protein altering variants were curated according to the ACMG guidelines for the interpretation of genetic variants [55] and the algorithm proposed by Guerreiro et al. [58] to determine pathogenicity (Additional file 1: Figures S14, S15 and Additional file 7: Supplementary methods).

Eleven deleterious variants were identified in the PSEN1 gene (Table 2 and Additional file 8: Table S6 and Additional file 9: Table S7). Three of these were novel; two classified as definite pathogenic, c. $485 \mathrm{~T}>\mathrm{G}$ (p.Ile162Ser) c.667C $>$ A (p.Gln223Lys); and one as probably pathogenic according to the Guerreiro algorithm, c.782C $>\mathrm{T}$ (p.Val261Ala). Four of these PSEN1 variants had been previously identified in the Colombian population c.349C $>$ G (p.Pro117Ala), c.428T $>C$ (p.Ile143Thr), c.839A $>$ C (p.Glu280Ala), and c.1247 T $>C$ (p.Ile416Thr) [38, 86-88], and four had been described in families outside Colombia with diverse ancestries c.356C $>$ T (p.Thr119Ile) [39], c.488A $>$ G (p.His163Arg) [89], c.791C $>\mathrm{T}$ (p.Pro264Leu) [89] and c.851C>T (p.Pro284Leu) [90]. PSEN1 c.839A >C (p.Glu280Ala) [86], of European origin, is the largest family in the world with familial Alzheimer's disease and living nearby is a family with the PSEN1 variant c.1247 T>C (p.Ile416Thr) [87] that originated in Africa.

PSEN1 c.782 T $>$ C (p.Val261Ala) was identified in a singlet without confirmed paternity, and it was classified as likely pathogenic (ACMG criteria)/probably pathogenic (Guerreiro) despite the lack of family history due to the report of three different pathogenic mutations in the same codon c.780G $>\mathrm{T}$ (p.Val261Phe) [91], c.780G $>\mathrm{A}$ (p.Val261Ile) [92], andc.780G $>C$ (p.Val261Leu) [93]. All the reported variants, except c.851C $>\mathrm{T}$ (p.Pro284Leu), presented as early-onset amnestic AD. The c.851C $>\mathrm{T}$ (p.Pro284Leu) carriers developed spastic paraparesis (SP), which is an atypical form of AD occasionally associated with certain PSEN1 mutations [91, 94, 95]. All the families with pathogenic PSEN1 mutations had autosomal dominant inheritance (Additional file 1: Figure S16); however, the singlet c.782 $\mathrm{T}>\mathrm{C}$ (p.Val261Ala) was indeterminate. Among these PSEN1 variants, six were of European origin, three were Native Americans, and one African (Table 2).

All the carriers of each variant, except c.791C $>\mathrm{T}$ (p.Pro264Leu), reported a known common ancestor (Additional file 1: Figure S16). Several families from the harbored the PSEN1 c.791C >T (p.Pro264Leu) variant, but we could not connect them by family history. Therefore, to prove that c.791C $>\mathrm{T}$ (p.Pro264Leu) was the result of a founder effect, we used the hap-IBD software to identify identical by descent (IBD) segments between the variant carrying chromosomes. All the PSEN1 c.791C>T (p.Pro264Leu) carrier haplotypes shared an IBD segment of $2.79 \mathrm{cM}$ around the PSEN1 locus, supporting the hypothesis of a common ancestor for all three families originating at about the same time (Additional file 1: Figure S17). PSEN1 c.791C $>\mathrm{T}$ (p.Pro264Leu) has been described in multiple populations (France [89, 96-99], UK [100, 101], Turkey [102], and Japan [103]) suggesting that PSEN1 c.791C $>$ T (p.Pro264Leu) is a recurring mutation. While the European carriers of this variant often present SP [104], this phenotype was not observed in the Colombian carriers of the variant. To determine if this 
Table 2 Pathogenic variants identified in disease causing genes

\begin{tabular}{|c|c|c|c|c|c|c|c|}
\hline \multicolumn{8}{|c|}{ Alzheimer disease genes } \\
\hline Gene & Coding change & dbSNP/ClinVar & ExAC & SIFT & Polyphen & CADD & Local ancestry \\
\hline$A P P$ & g.(26253828_30011000)dup & SCV001751549 & . & - & - & - & European \\
\hline \multirow[t]{11}{*}{ PSEN1 } & c.349C>G (p.Pro117Ala) & rs63750550 & . & $\mathrm{D}$ & $P$ & 26.9 & European \\
\hline & c.356C>T (p.Thr119\|le) & rs1566630791 & & $\mathrm{T}$ & $P$ & 24.4 & European \\
\hline & c.428T>C (p.lle143Thr) & rs63750004 & . & D & $\mathrm{D}$ & 26.8 & European \\
\hline & c.485T>G (p.lle162Ser) & rs1898533739 & . & $\mathrm{D}$ & $\mathrm{D}$ & 32 & Native American \\
\hline & c.488A>G (p.His163Arg) & rs63750590 & & $\mathrm{T}$ & B & 23.4 & European \\
\hline & c.667C >A (p.GIn223Lys) & rs1898776259 & & $\mathrm{D}$ & $\mathrm{D}$ & 33 & Native American \\
\hline & c.782T>C (p.Val261Ala) & SCV001751539 & . & D & $P$ & 25.9 & Undetermined \\
\hline & c.791C>T (p.Pro264Leu) & rs63750301 & . & $\mathrm{D}$ & $\mathrm{D}$ & 35 & Native American \\
\hline & c.839A >C (p.Glu280Ala) & rs63750231 & . & $\mathrm{D}$ & $\mathrm{D}$ & 29.3 & European \\
\hline & c.851C>T (p.Pro284Leu) & rs63750863 & & D & $\mathrm{D}$ & 33 & European \\
\hline & c.1247T>C (p.lle416Thr) & SCV001751540 & . & $\mathrm{D}$ & $P$ & 25.9 & African \\
\hline PSEN2 & c.487C>T (p.Arg163Cys) & rs200931244 & & D & D & 35 & African \\
\hline \multicolumn{8}{|l|}{ FTLD genes } \\
\hline Gene & Coding change & dbSNP & ExAC & SIFT & Polyphen & CADD & Local ancestry \\
\hline C9ORF72 & (GGGGCC)n Repeat Expansion & rs143561967 & & . & . & . & European \\
\hline GRN & c.709-2A>G (p.Ala237fs) & rs63750548 & . & . & . & 23.1 & European \\
\hline \multirow[t]{2}{*}{ MAPT } & c.902C>T (p.Pro301 Leu) & rs63751273 & . & D & D & 34 & European \\
\hline & c.1189c>T (p.Pro397Ser) & rs1295855402 & & D & $\mathrm{D}$ & 25 & European \\
\hline \multirow[t]{2}{*}{ TARDBP } & c.881G>T (p.Gly294Val) & rs80356721 & 0.00000824 & $\mathrm{~T}$ & $P$ & 18.89 & European \\
\hline & c.1147A>G (p.lle383Val) & rs 80356740 & 0.00000865 & $\mathrm{~T}$ & B & 0.308 & European \\
\hline \multirow[t]{2}{*}{ TBK1 } & c.1257_1258del (p.Val421Cfs*26) & rs1392685429 & . & . & . & & European \\
\hline & c.1717C>T (p.Arg573Cys) ${ }^{+}$ & rs772820487 & 0.00003329 & $\mathrm{~T}$ & $\mathrm{D}$ & 29.6 & European \\
\hline \multicolumn{8}{|l|}{ ALS genes } \\
\hline Gene & Coding change & dbSNP & ExAC & SIFT & Polyphen & CADD & Local ancestry \\
\hline ANXA11 & c.904C>T (p.Arg302Cys) & rs142183550 & 0.0000412 & D & D & 31 & Native American \\
\hline FIG4 & c.122T>C (p.lle41Thr) ${ }^{+}$ & rs121908287 & 0.001 & $\mathrm{D}$ & D & 26.5 & European \\
\hline HNRNPA2B1 & c.965G>A (p.Gly322Glu) & SCV001751542 & . & D & $\mathrm{D}$ & 23.6 & Native American \\
\hline SOD1 & c.63C>G (p.Phe21Leu) & rs1555836170 & . & $\mathrm{T}$ & $\mathrm{D}$ & 22.9 & Native American \\
\hline SQSTM1 & c.1175C>T(p.Pro392Leu) & rs104893941 & 0.0009 & D & B & 34 & European \\
\hline TUBA4A & c.820C > G (p.Pro274Ala) & rs1241875438 & & & $\mathrm{D}$ & 23.8 & Native American \\
\hline TUBB4A & c.811G>A (p.Ala271Thr) & rs587777074 & 0.000003992 & & $P$ & 22.8 & Native American \\
\hline UBQLN2 & c.724G >A (p.Ala242Thr) & SCV001751543 & . & D & $\mathrm{D}$ & 25.9 & Undetermined \\
\hline \multicolumn{8}{|c|}{ Other neurodegeneration associated genes } \\
\hline Gene & Coding change & dbSNP & ExAC & SIFT & Polyphen & CADD & Local ancestry \\
\hline CSF1R & c.2068G>A (p.Gly690Ser) & rs141866247 & 0.0000165 & $\mathrm{~T}$ & $\mathrm{D}$ & 23.1 & Native American \\
\hline DNAJC5 & c.347T>G (p.Leu116Arg) & SCV001751544 & . & $\mathrm{D}$ & P & 27.2 & African \\
\hline LRRK2 & c.4334C>G (p.Ser1445Cys) & rs1945001552 & & $\mathrm{T}$ & $P$ & 24.3 & European \\
\hline
\end{tabular}

ExAC ExAC database minor allelic frequency. SIFT scores are D, deleterious, and T, tolerated. PolyPhen-2 scores are D, probably damaging, $\mathrm{P}$, possibly damaging, and B, benign. CADD corresponds to the Phred score. Variants with + were identified in homozygous states. GenBank transcripts for each gene can be found in Additional file 4: Table S3

phenotypic heterogeneity is related to the ancestral haplotype wherein the variant arose, we used RFMix to estimate the ancestry of the variant carrier haplotype (Table 2 and S6). In the TANGL cohort, PSEN1 c.791C > T (p.Pro264Leu) resided on a Native American haplotype, which suggests that the haplotype of origin may play a role in the different expressivity and clinical manifestations between the variant carriers. Six of the other pathogenic PSEN1 variants resided on European haplotypes, two variants were present in Native American and one in an African background. The multiancestral origins of the PSEN1 variants suggest that the admixture process contributed to the introduction of pathogenic variants to a population. 
Two of the PSEN1 variants described in this cohort had been previously identified in other families in Colombia [c.428T >C (p.Ile143Thr) [38], c.356C >T (p.Thr119lle)], and in Argentina [c.356C $>\mathrm{T}$ (p.Thr119Ile) [39]]. We performed additional array genotyping to test for IBD between the members of these families and those from the TANGL cohort. The Colombian carriers of c.428T $>C$ (p.Ile143Thr) and c.356C > T (p.Thr119Ile) showed IBD overlapping the PSEN1 locus (Additional file 1: Figures S18 and S19). Interestingly, the Colombian individuals who harbored c.356C $>\mathrm{T}$ (p.Thr119Ile) with whom no shared ancestor could be determined by history carried a small IBD segment shared with the Argentinian carrier of the same variant (Additional file 1: Figure S20). The geographical expanse over which these variants reside could reveal small population migratory streams from Europe or within the South American continent.

In addition to the eleven pathogenic variants, we identified four benign variants in PSEN1. c.1279A $>$ G (p.Ile427Val) and c.114C >A (p.His38Gln) that did not segregate with the illness, while c.118G $>$ A (p.Asp40Asn) and c.953A $>\mathrm{G}$ (p.Glu318Gly) have been reported in cases and controls without a clear disease association [105107]. Thus, most of the PSEN1 missense variants in this cohort are pathogenic and have an age-dependent phenotype of amnestic AD. In contrast, the majority of the variants observed in PSEN2 were either benign or had been previously classified as risk factors for AD. Only the variant c.487C $>\mathrm{T}$ (p.Arg163Cys), which had been described in a Chinese patient with AD [108], was classified as likely pathogenic (Additional file 1: Figure S21). Interestingly, this variant resided on an African haplotype in the Colombian carrier. No pathogenic variants were observed in APP; but one individual with AD had copy number variation (CNV) spanning APP [104] (chromosome 21 g.(26253828_30011000)dup, Additional file 1: Figure S22). These results confirm PSEN1 as the most prevalent gene associated with genetic AD in our cohort, mostly as the result of founder effects, and that the current genetic burden of the TANGL cohort is influenced by the genetic diversity of its founders.

\section{Variants in FTLD-MND associated genes}

We performed the same curation process for FTLDMND associated genes (MAPT, C9ORF72, GRN, VCP, FUS, CHMP2B, TBK1, TARDBP). Most of the individuals with genetic forms of FTLD-MND in the TANGL cohort had deleterious variants in MAPT and TARDBP (Table 2 and Additional file 8: Table S6 and Additional file 9: Table S7). The MAPT c.1189C>T (p.Pro397Ser) variant was identified in three independent families from the same geographic region that shared IDB segment of $2.89 \mathrm{cM}$ overlapping the locus (Additional file 1 :
Figures S23 and S24). This variant had been previously reported in five apparently unrelated Spanish families [42], and like the Spanish counterpart, the Colombian $M A P T$ c. $1189 \mathrm{C}>\mathrm{T}$ (p.Pro397Ser) carriers had variable expressivity of the illness (Additional file 9: Table S7 and Additional file 10: Table S8). To elucidate whether the Colombian MAPT c.1189C>T (p.Pro397Ser) carriers were IBD with the Spanish families, we used exome sequencing data from a Spanish patient to search for similarities in the variant carrying haplotype. We identified a minimal shared haplotype of $2.65 \mathrm{cM}$ including the $M A P T$ locus, which suggests that the Colombian families share a common ancestor with the Spanish carriers of MAPT c.1189C >T (p.Pro397Ser) (Additional file 1: Figure S25).

Two siblings with FTLD-MND born of consanguineous parents were homozygous for the TBK1 c.1717C $>\mathrm{T}$ (p.Arg573Cys) variant (Additional file 1: Figure S26). Haploinsufficiency of TBK1 has been previously associated with familial ALS and FLTD and is a known mechanism of pathogenicity [109]. Homozygosity of nonsense $T B K 1$ variants has been proven to be lethal in mice [110]. A second variant in TBK1 was c.1257_1258del (p.Val421Cfs*26), identified in two unrelated individuals that shared an IBD segment of $3.1 \mathrm{cM}$ including the $T B K 1$ locus (Additional file 1: Figure S27). We identified two variants in TARDBP that had been previously reported in European populations with diagnosis of ALS $[111,112]$, and in contrast with these cohorts, Colombian TARDBP c.1147A>G (p.Ile383Val) carriers had significant intra-familial variability with heterogeneous FTLDMND spectrum disorders (Additional file 1: Figure S28). Our study identified only one carrier of C9ORF72 expansion, a single carrier of a pathogenic variant in $G R N$ (Additional file 1: Figure S29), and no disease-causing variants in $C H M P 2 B$, FUS, or $V C P$. While the frequency of the identified mutations differs from those reported in European descent cohorts $[59,113]$, all the identified pathogenic variants in these FTLD-MND associated genes resided on European haplotypes.

\section{Other genes associated with ALS in the cohort}

To explore the phenotypic and genetic overlap between FTLD and ALS, we searched for deleterious variants in nineteen additional genes associated with ALS, with or without FTLD (Additional file 1: Figure S14, S15 and Additional file 7: Supplementary methods). The SQSTM1 [MIM: 601530] c.1175C >T (p.Pro392Leu) variant was present in 11 unrelated cases and two controls of the TANGL cohort. These cases were unrelated and were clinically heterogeneous: six had diagnosis of $\mathrm{AD}$, three of FTLD, one of CBD, and one PSP (Table 2 and Additional file 8: Table S6). Eight of the eleven cases had 
family history of dementia or neurodegenerative disease, and none of them carried other pathogenic mutations in the explored disease-causing genes. This variant was initially reported in European individuals with familiar forms of FTLD, Paget's disease of the bone, and ALS [114-116]. Later studies identified this variant both in cases and controls, suggesting that it may be a risk factor rather than causal for illness $[117,118]$.

The SQSTM1 c.1175C>T (p.Pro392Leu) is the result of founder effects in Belgian, Dutch, and Spanish individuals [119], and it was present in five individuals from the European cohort of the 1000GP. We used HAP-IBD to search for IBD between the Colombian and the 1000GP carriers of SQSTM1 c.1175C >T (p.Pro392Leu). Ten carriers of the TANGL cohort shared IBD segments $>2 \mathrm{cM}$ overlapping the variant, which resided in a European haplotype as well (Additional file 1: Figure S30). To determine IBD at a smaller scale, we did a manual alignment of all the variant-carrying haplotypes and detected an IBD segment of $\sim 1 \mathrm{cM}$ between all the TANGL cohort and 1000GP European SQSTM1 c.1175C>T (p.Pro392Leu) carriers (Additional file 1: Figure S31). This observation suggests that SQSTM1 c.1175C >T (p.Pro392Leu) shows the signature of a founder effect that pre-dates the Spanish invasion. Variants with higher allelic frequency also show IBD between the TANGL cohort and with other carriers outside of Colombia.

In contrast to the pathogenic variants in the FTLDMND associated genes, five of the eight disease associated variants identified in the ALS panel were of Native American origin while only two were of European ancestry (Table 2). However, most of these individuals with pathogenic and likely pathogenic variants in Native American haplotypes presented with FTLD phenotypes (Additional file 8: Table S6 and Additional file 9: Table S7). For example, the TUBA4A [MIM: 191110] c.820C $>$ G (p.Pro274Ala) variant was identified in two independent families with positive family histories of dementia and diagnosis of bvFTD and EOD without motor neuron disease (Additional file 1: Figure S32). As described previously for other variants, these families shared a long IBD haplotype of $15.54 \mathrm{cM}$ overlapping the locus, suggesting a recent common ancestor (Additional file 1: Figure S33). The SOD1 [MIM: 147450] c.63C>G (p.Phe21Leu) variant was identified in one patient with sporadic navPPA who did not have any motor or ALSassociated symptoms. This variant and others in this same amino acid [c.62 T>G (p.Phe21Cys)] had been previously reported in patients with ALS [120, 121]. Additional likely pathogenic variants in ANXA11 [MIM: 602572] and HNRNPA2B1 [MIM: 600124] residing in Native American haplotypes were identified in patients with SvPPA and bvFTD. These results further intertwine
ALS and FTLD with several genes previously associated exclusively with ALS that may also be responsible for a FTLD phenotype in a different ancestral context. The genetic and clinical heterogeneity of ALS associated genes had been previously described in European population [122], but the inclusion of diverse individuals expands the extent of genetic overlap between FTLD and ALS.

A patient with PSP was homozygous by descent for a European haplotype harboring the FIG4 [MIM: 609390], c.122 T>C (p.Ile41Thr). Although this gene has been associated with autosomal dominant forms of ALS, this same specific variant has been reported in compound heterozygosity with nonsense variants in European individuals with autosomal recessive cases of CharcotMarie-Tooth's disease [123] [MIM: 611228]. A family presenting with FTLD-ALS was shown to have a novel c.724G $>$ A (p.Ala242Thr) variant in UBQLN2 [MIM: 300264]. UBQLN2, found on the X-chromosome, is associated with ALS or FTLD-MND, with a lower penetrance in females [124]. The family with this mutation had late onset bvFTD presentation in the female carrier, while the male carrier had FTLD-MND (Additional file 1: Figure S34).

\section{Other genes associated with neurodegenerative disorders}

Several families with EOD were explained by variants in other non-AD-FTD-ALS genes (Additional file 1: Figures S14 and S15). A family with an unspecified autosomal dominant EOD had a novel mutation in DNAJC5 [MIM: 611203] c.347 T>G (p.Leu116Arg) residing on an African haplotype. Their phenotype and postmortem brain tissue histopathology was compatible with adultonset ceroid neuronal lipofuscinosis-4B (CNL4B) [MIM: 162350] (Additional file 1: Figure S35). A novel likely pathogenic variant in LRRK2 [MIM: 609007] c.4334C>G (p.Ser1445Cys) was identified in a patient with a European background and non-motor symptoms in Parkinson's disease and dementia. One patient with a family history of cancer and dementia carried the CSF1R [MIM: 164770] c.2068G $>$ A (p.Gly690Ser) variant in a Native American haplotype. CSF1R mutations have been associated with Hereditary Diffuse Leukoencephalopathy with Spheroids (HDLS) [125] [MIM: 221820] A postmortem brain tissue examination supported HDLS diagnosis for the CSF1R c.2068G>A (p.Gly690Ser) variant carrier (Additional file 1: Figure S36). These families provide novel insights on genetic-phenotypic relationships.

Despite an extensive evaluation of known genes previously reported for Mendelian forms of dementia, we were not able to identify a disease-causing variant in all families with autosomal dominant inheritance of the illness. Of the 566 families included in the present study, 
59 had autosomal dominant inheritance defined as three or more affected individuals in two consecutive generations (Additional file 11: Table S9). For the 18 families in which all individuals had early onset of symptoms (<65 years), we could identify disease causing variants in all but three, and 13 of them carried pathogenic PSEN1 variants. In families with both early and late onset cases, we identified disease causing variants in seven of 33. No disease-causing variant was identified in the 12 individuals from the eight families where everyone had late onset, but 10 of them carried at least one $A P O E$ [MIM: 107741] $€ 4$ allele (two were $A P O E \in 3 / \epsilon 3$, six were $€ 3 / \epsilon 4$, and four $\epsilon 4 / \epsilon 4)$. In conclusion, a pathogenic or likely pathogenic variant was identifiable in the families with autosomal dominant inheritance in which most of the affected individuals had disease onset before 65 years.

\section{Genetic variation associated with $A D$ risk genes}

Both rare and common variants can have a small effect size on AD risk [126]. To explore rare variants conferring intermediate risk for the illness, we selected three genes (TREM2, SORL1, and ABCA7) that have shown odds ratio (OR) higher than two $(\mathrm{OR}>2)$ in disease association studies [69] Using the criteria suggested by Bellenguez et al. [69], we identified 14 protein truncating variants (PTV) and 16 strictly damaging (SD) variants in TREM2, SORL1, and ABCA7 (Table 3 and Additional file 12: Table S10).

The most common risk-conferring variants in the TANGL cohort resided on TREM2, with over a hundred individuals carrying SD or PVT in this gene (Additional file 12: Table S10). The most prevalent variant was c.469C $>$ T (p.His157Tyr), with 50 heterozygous and seven homozygous carriers. All the c.469C $>\mathrm{T}$ (p.His157Tyr) carriers were IBD for a Native American haplotype. Two out of three algorithms classified His157Tyr as definitely pathogenic, while a meta-analysis determined TREM2 c.469C $>$ T (p.His157Tyr) has an OR $=3.65$ [127], and therefore, it qualified for the present study. Additionally, we identified 33 TREM2 c.140G>A (p.Arg47His) carriers in our cohort; three of them were homozygous for this variant (Additional file 12: Table S10). All the TREM2 c.140G >A (p.Arg47His) carriers from the TANGL cohort shared an IBD European haplotype overlapping the TREM2 locus, and this same variant-carrying haplotype was present in five European individuals from the 1000GP who showed IBD with the Colombian carriers (Additional file 1: Figure S37). Besides risk conferring variants in Native American and European haplotypes, an African TREM2 haplotype [GenBank: NM_001271821] carrying c.572G $>$ A (p.Trp191*), c.632T $>$ C (p.Leu211Pro), and c.287C $>$ A (p.Thr96Lys) was identified in 10 individuals. This haplotype was previously associated with an increased risk in African-American cohorts [128]. Unlike the previous cases of homozygosity, one individual with early-onset $\mathrm{AD}$ was a compound heterozygote with both the Thr96Lys/Trp191\%/Leu211Pro haplotype and the c.469C $>$ T (p.His157Tyr) variant, suggesting that genetic risk factors from different ancestral origins may coexist in admixed individuals and populations.

Rare variants in TREM2 are population specific. For example, TREM2 c.140G $>$ A (p.Arg47His) is associated with increased risk for AD in European descent populations [129, 130] but not in African [128] or Asian [131, 132], while TREM2 c.469C $>\mathrm{T}$ (p.His157Tyr) shows association with AD in Asian [127, 133] but not in European [134] or African [128] cohorts. Interestingly, the c.469C $>$ T (p.His157Tyr) variant was found in Colombia on a Native American haplotype, raising the possibility that this allele arrived from Asia to the American continent close to the time when the Americas were first populated 15,000-20,000years ago. To support this hypothesis, we searched for this variant in the Human Genome Dating database [135], which uses coalescent modeling to estimate the time to the most recent common ancestor (TMRCA) between the variant carriers and the age of the variant. The estimated age of the c.469C $>\mathrm{T}$ (p.His157Tyr) allele is 1265 generations (95\% confidence interval of 1108.5-1430.9), which corresponds to 31,625 years by setting one generation equivalent to 25 years (https://human.genome.dating/snp/rs2234255). In contrast, the c.140G $>$ A (p.Arg47His) variant emerged more recently, as it was estimated to be 425 generations old or 10,625 years (https://human.genome.dating/snp/ rs75932628), dating to a time before gene flow from Europe to the Americas occurred. These results lead us to conclude that the disease burden in this population is not only affected by the recent admixture after the conquest of the Americas, but was also affected by migrations [136] during the original populating of the continent.

Risk-conferring variants in $A B C A 7$ and SORL1 were less prevalent than those in TREM2. Most of the variants detected in $A B C A 7$ consisted in PTV and resided on African haplotypes (Additional file 1: Figure S37). The majority in SORL1 were SD variants of European origin, two homozygous carriers of $A B C A 7$ variants c.2124_2130del (p.Glu709fs) and c.4886C $>\mathrm{T}$ (p.Ser1629Leu), and a compound heterozygote of risk variants from different ancestral origins. There were no compound heterozygous or homozygous variants for SORL1, and the c.6550G >A (p.Ala2184Thr) variant was only found in a healthy centenarian. Additionally, a search for risk associated variants in $A D A M 10$ [77, 78], identified c.510G >C (p.Gln170His) in ten individuals, including one homozygous patient. These reported variants in TREM2, SORL1, $A B C A 7$, and $A D A M 10$ were IBD in carriers of the same 
Table 3 Variants in risk-associated genes

\begin{tabular}{|c|c|c|c|c|c|c|c|}
\hline Gene & Coding change & dbSNP/ClinVar & Classification & ExAC & $1000 \mathrm{G}$ & CADD & Local ancestry \\
\hline \multirow[t]{17}{*}{$A B C A 7$} & $c .2 T>C$ & rs1347920426 & PTV (nonsense) & . & . & 24.9 & Native American \\
\hline & c.236A>C (p.Asn79Thr) & rs377401443 & SD & 4.16E-05 & & 24.5 & African \\
\hline & c.1180_1190del (p.Leu396fs) & rs567222111 & PTV (frameshift) & 0.0005 & 0.0022 & . & African \\
\hline & c.1531G>T (p.Glu511*) & rs374932832 & PTV (nonsense) & $7.60 \mathrm{E}-05$ & . & 39 & African \\
\hline & c.1776G>T (p.Trp592Cys) & SCV001751545 & SD & . & . & 26 & African \\
\hline & c.2124_2130del (p.Glu709fs)+ & rs547447016 & PTV (frameshift) & 0.0024 & 0.0006 & & European \\
\hline & c. $2194 C>T\left(p . G \ln 732^{*}\right)$ & rs1030634619 & PTV (nonsense) & . & . & 36 & European \\
\hline & c.2552+11_2552+58del & rs1178315251 & PTV (splice) & . & . & . & African \\
\hline & c.2611G>C (p.Asp871His) & rs139251928 & SD & 0.0004 & 0.0014 & 24.8 & African \\
\hline & c.3781 delC (p.Pro1261fs) & SCV001751546 & PTV (frameshift) & & & . & Native American \\
\hline & c.4208delT (p.Leu1403fs) & rs538591288 & PTV (frameshift) & 0.0011 & . & . & European \\
\hline & c.4465C>T (p.Arg1489*) & rs753664323 & PTV (nonsense) & $6.66 \mathrm{E}-05$ & . & 39 & European \\
\hline & c.4886C>T (p.Ser1629Leu)+ & rs 184590335 & SD & 0.0012 & 0.0006 & 35 & Native American \\
\hline & c.4895C>T (p.Pro1632Leu) & rs 143083561 & SD & 0.0002 & 0.0006 & 34 & African \\
\hline & c.5302delC (p.Leu1768fs) & rs1348650979 & PTV (frameshift) & . & . & . & Native American \\
\hline & c. $5463+2 T>C$ & rs374611445 & PTV (splice) & $2.81 \mathrm{E}-05$ & . & 23.7 & European \\
\hline & c.5794C>T (p.Arg1932C) & rs114787084 & SD & 0.0002 & 0.0006 & 34 & African \\
\hline \multirow[t]{9}{*}{ SORL1 } & c.994C>T (p.Arg332Trp) & rs772110877 & SD & 5.77E-05 & . & 35 & European \\
\hline & c.1432G>C (p.Ala478Pro) & SCV001751547 & SD & . & . & 28.2 & European \\
\hline & c.1496C>T (p.Ser499Leu) & rs764032259 & SD & $8.24 \mathrm{E}-06$ & . & 35 & European \\
\hline & c.2200G >A (p.Asp734Asn) & rs 148430425 & SD & 0.0011 & . & 34 & European \\
\hline & c. $2230 \mathrm{C}>\mathrm{T}\left(\mathrm{p} . \operatorname{Arg} 744^{*}\right)$ & rs 1050845490 & PTV (nonsense) & . & . & 39 & European \\
\hline & c.2710C >T (p.Arg904Trp) & rs148966249 & SD & 4.12E-05 & $2.00 \mathrm{E}-04$ & 33 & Native American \\
\hline & c.3679G>T (p.Gly1227Cys) & rs1765488318 & SD & & & 34 & European \\
\hline & c.4520C>T (p.Pro1507Leu) & rs1308522330 & SD & & . & 26.2 & Undetermined \\
\hline & c.6550G >A (p.Ala2184Thr) & rs369618646 & SD & 4.16E-05 & . & 34 & African \\
\hline \multirow[t]{4}{*}{ TREM2 } & c.140G >A (p.Arg47His)+ & rs75932628 & SD & 0.0021 & 0.002 & 33 & European \\
\hline & c.469C>T (p.His157Tyr)+ & rs2234255 & SD & 0.0036 & 0.0028 & 23.1 & Native American \\
\hline & $\begin{array}{l}\text { NM_001271821 } \\
\text { c.287C >A (p.Thr96Lys) } \\
\text { c.572G>A(p.Trp191*) } \\
\text { c.632T>C (p.Leu211Pro) }\end{array}$ & $\begin{array}{l}\text { rs2234253 } \\
\text { rs2234258 } \\
\text { rs2234256 }\end{array}$ & PTV (nonsense) & & & & African \\
\hline & c.594G>A (p.Trp198*) & rs 1765488318 & PTV (nonsense) & . & . & 39 & Undetermined \\
\hline ADAM10 & c.510G >C (p.Gln170His)+ & rs61751103 & SD & 0.0012 & 0.0012 & 19.17 & European \\
\hline
\end{tabular}

PTV protein truncating variant, SD strictly damaging, ExAC ExAC database minor allelic frequency. CADD corresponds to the Phred score. Variants denoted with a + were identified in homozygous states. GenBank transcripts for each gene can be found in Additional file 4: Table S3

variant (Additional file 1: Figures S37, S38, S39 and S40). In summary, the characteristics we described for diseasecausing variants such as IBD between carriers, multiple ancestral origins of deleterious variants within the same gene, and autozygosity were present in variants with higher allelic frequencies in risk-associated genes.

The high allelic frequency of some risk conferring variants in the TANGL cohort allowed the detection of individuals who were homozygous by descent and raised the hypothesis of consanguinity between their parents, as was the case for the two families with recessive dementias [TBK1 c.1717C $>\mathrm{T}$ (p.Arg573Cys) and FIG4 c.122 T>C (p.Ile41Thr)]. We used Hap-IBD and manual haplotype alignment to estimate the autozygosity of the homozygous individual for risk-associated variants in $A B C A 7$ [c.2124_2130del (p.Glu709fs) and c.4886C $>\mathrm{T}$ (p.Ser1629Leu)], TREM2 [c.140G >A (p.Arg47His) and c.469C $>\mathrm{T}$ (p.His157Tyr)] and ADAM10 [c.510G $>\mathrm{C}$ (p.Gln170His)]. Five individuals from three families who were the offspring of related parent had autozygous segments $>30 \mathrm{cM}$ overlapping the risk associated variant (Additional file 13: Table S11). The remaining individuals had smaller autozygous segments, suggesting background relatedness of the population due to a small effective population size or bottlenecks $[137,138]$. 


\section{Discussion}

Genetic drift has been one of the main forces shaping human genomic variation $[139,140]$. While populations that emerge from a bottleneck will harbor reduced genetic variation, over time, such a population can accumulate higher numbers of deleterious variants due to random fluctuations in allele frequencies [141]. Furthermore, deleterious allele frequencies decrease more slowly in smaller populations because natural selection acts on fitness differences and therefore requires genetic variation [141]. The Colombian tri-continental admixture among the Native Americans, Europeans, and Africans combined a portion of the genetic disease burden that was previously limited to each of these ancestral populations. Within the backdrop of an admixed population, numerous infectious diseases extracted a very steep mortality. As a consequence, the small isolated settlements that survived the bottleneck rapidly expanded locally during the colonial period [1]. These multiple isolated bottlenecks each with their own rare variants added to the diversity over the entire population. The TANGL cohort recapitulated the admixture patterns previously described in the Colombian population, suggesting that the country's demographic history is likely to underlie the modern clustering of familial neurodegenerative diseases arising from multi-ancestral rare disease-associated alleles.

In this cohort, most familial early-onset AD cases were caused by variation in the PSEN1 gene. We identified eleven different pathogenic PSEN1 variants from multiple ancestral origins, nearly all attributed to founder effects. The PSEN1 mutations emerged from a small effective population in each of the early settlements that constituted a patchwork of bottlenecks dispersed throughout the country. Because people tended to remain geographically isolated, the rare variants represent a local genetic footprint. Survivors who emerged from the bottleneck had escaped the large number of infectious diseases responsible for decimating the population. During the historical period of colonization, populations in these settlements grew rapidly as the incidence of diseases diminished, which favored the segregation of potentially damaging variants at higher rates. The question arises as to whether the PSEN1 mutations could be under positive selection or are the mutations completely explained by drift. Because PSEN1 mutant phenotypes do not appear until after the age of child-bearing, it is unnecessary to invoke trade-off effects for maintaining the mutation in the population. Positive selection for Alzheimer risk in the context of infectious burden has been previously attributed to the $A P O E \in 4$ risk allele [142]. PSEN1 mutations cause the production of excess amyloid-beta, which may function as an anti-microbial peptide (AMP) [143].
In this manner, PSEN1 mutations may have been positively selected as protection against the enormous mortality of infectious diseases. AMPs function as an ancient component of the innate immune system that target bacteria, mycobacteria, enveloped viruses, fungi, and protozoans [144]. Amyloid beta is active against at least eight common and clinically relevant microorganisms, and several anti-amyloid-beta clinical trials have reported increased rate of infections among the participants [143, $145]$. However, given the short $\sim 500$-year interval since the selective pressure occurred and the $\sim 100$-year pulselike nature of the selection, the possibility of positive selection must remain speculative. Without a sufficient time interval for the mutation to spread widely through the population, the only indirect support for positive selection might consider the collective fitness conferred by all of the PSEN1 mutations due to their shared phenotypic effect of increasing amyloid beta as an AMP. Whether these mutations represent a statistical excess will require further study, but given the population size at the time to which the mutations can be historically traced (see ancestry data for each mutation), it is likely that the mutations derived from a small effective population, thus supporting their possible over-representation. A comparison comes from large catchment groups for clinics with an interest in familial dementias-one in Alabama had no PSEN1 cases in their series [146] and another in San Francisco had six PSEN1 cases (personal communication, Jennifer Yokoyama, University of California San Francisco). In one study that sought early-onset Alzheimer patients from 28 university hospitals across France spanning the dates 1993 to 2016, 17 sporadic cases carried a PSEN1 mutation [104]. However, any comparison with our cases is problematic because ten of these arose de novo, which was not the case in the TANGL cohort, and some were of unknown pathogenicity.

In addition to the PSEN1 variants, we identified multiple rare variants causing autosomal dominant early-onset dementia. Variants were usually found in one locality and likely derived from a common ancestor (Additional file 1: Figure S41). Previous studies had reported disease causing variants for other neurological disorders with the signature of founder effects; among these are four different cerebral autosomal dominant arteriopathy with subcortical infarcts and leukoencephalopathy (CADASIL) [MIM: 125310] associated variants in NOTCH3 [MIM: 600276, c.307C $>\mathrm{T}$ (p.Arg103Cys), c.421C $>\mathrm{T} \quad$ (p.Arg141Cys), c.484 T >A (p.Cys162Ser), c.1363 T>C (p.Cys455Arg)] [147, 148], a familial episodic pain syndrome [MIM: 615040] with a variant in TRPA1 [MIM: 604775, c.2564A>G (p.Asn855Ser)] [149], Huntington's disease [150] [MIM: 143100], a Parkinson disease variant in LRRK2 [c.6055G>A (p.Gly2019Ser)] 
[151], blepharophimosis-ptosis-epicanthus inversus syndrome (BPES) [MIM: 110100] type 1 with a FOXL2 [MIM: 605597, c.157C > T (p.Gln53*)] variant and BPES type 2 with FOXL2 in-frame 30 bp duplication (c. 909938dup) [152], a complex ataxia due to a KIF1A variant [MIM: 601255, variant c.304G>C (p.Gly102Arg)], generalized epilepsy with febrile seizures plus (GEFS+) [MIM: 604403] with SCN1A [MIM: 182389 c.5225A >G (p.Asp1742Gly)] variant [153], and non-syndromic hearing loss [MIM: 220290] due to a GJB2 variant [MIM: 121011 c.35delG (p.Gly12Valfs*] [154] . Founder effects can also be detected in other non-neurologic conditions: BRCA1/2 variants [MIM: 113705, 600185] among Colombian women with breast and ovary cancer increased the prevalence of these variants in the studied population [155]. Most of these mutations map to small distinct locales that when, taken together, demonstrate the remarkable overlap of the genetic and geographic maps.

This study underscores the numerous genetic insights that can emerge from Latin American populations. Another example is the putative modifier gene-homozygosity of the Christchurch variant in ApoE3-that may strongly delay the onset of Alzheimer's disease [156]. This gene variant and many of the rare large effect size mutations reported here arose due to the unique genetic history of the region. Ongoing interest in Latin American genetic studies, akin to all genetic studies in underrepresented populations, must consider the ethical implications of the research. Over the many years these were obtained, the research was conducted with the full involvement of the community and extensive interactions with and informed consent from the contributing families.

\section{Conclusions}

Demographic history plays a significant role in shaping a population's genetic risk for disease. The genetic complexity of the dementias offers a phenotypic heading for a search to uncover genetic variation for the familial dementias. In the Colombian population, founder effects led to a large number of ancestral disease-causing alleles from each of three admixed continents. We also observed a confluence of rare variants arising from different ancestral origins in dementia risk-conferring genes. Variants of different ancestries combined to create a heterogeneous landscape for the genetic risk of dementia. In addition to the significant role of admixture and drift, we raise the question of whether positive selection of PSEN1 mutations could contribute to the large number of these in a relatively small effective population size. PSEN1 variants lead to excess of amyloid-beta, which may function as anti-microbial protein and may have protected against the massive mortality due to infectious diseases during the conquest and colonization of the Americas. This work reinforces the need to include diverse populations for gene-trait association studies including populations that underwent bottlenecks as a source for gene discovery.

\begin{abstract}
Abbreviations
1000GP: 1000 Genomes Project; ACMG: American College of Medical Genetics; AD: Alzheimer's disease; AFR: African; ALS: Amyotrophic lateral sclerosis; AMP: Anti-microbial peptide; BPES: Blepharophimosis-ptosis-epicanthus inversus syndrome; bvFTD: Behavioral variant of frontotemporal dementia; CADASIL: Cerebral autosomal dominant arteriopathy with subcortical infarcts and leukoencephalopathy; CBD: Cortico-basal degeneration; CDR: Clinical Dementia Rating; CLM: Colombians from Medellín; CNL4B: Ceroid neuronal lipofuscinosis-4B; CNV: Copy number variation; CV: Cross validation; DNA: Deoxyribonucleic acid; EOD: Early-onset dementia not otherwise specified; EUR: European; FTLD: Frontotemporal lobar degeneration; GEFS+: Generalized epilepsy with febrile seizures plus; GWAS: Genome wide association studies; HDLS: Hereditary diffuse leukoencephalopathy with spheroids; IBD: Identity by descent; LD: Linkage disequilibrium; IvPPA: Logopenic variant of primary progressive aphasia; MAF: Minor allele frequency; MND: Motor neuron disease; NAT: Native American; navPPA: Non-fluent/agrammatic variant of primary progressive aphasia; OMIM: Online Mendelian Inheritance in Men database; OR: Odds ratio; PC: Principal component; PCA: Principal component analysis; PSP: Progressive supranuclear palsy; PTV: Protein truncating variants; SD: Strictly damaging; SNVs: Single nucleotide variants; SP: Spastic paraparesis; SVPPA: Semantic variant of primary progressive aphasia; TANGL: The Admixture and Neurodegeneration Genomic Landscape; TMRCA: Time to the most recent common ancestor; WGS: Whole genome sequencing.
\end{abstract}

\section{Supplementary Information}

The online version contains supplementary material available at https://doi. org/10.1186/s13073-022-01035-9.

Additional file 1: Figure S1. Demographic information of the TANGL cohort and the Colombian population. Figure S2. Pipeline for whole genome sequence data quality control (QC). Figure S3. Principal Component Analysis of whole genomes from 1000 Genomes project and the TANGL cohort. Figure S4. Cross validation error for unsupervised ADMIXTURE clustering analysis of the TANGL cohort probands. Figure S5. Cross Validation Error for unsupervised ADMIXTURE clustering of the multiancestral dataset (TANGL genomes with the European and African populations from the 1000GP and Native American genomes from Mao et al. Figure S6. Global ancestry proportions of the TANGL cohort calculated by ADMIXTURE and sum of RFMix local ancestry estimation. Figure S7. Correlation of global ancestry proportions calculated for each individual by two different software, RFMix sum of local ancestries vs ADMIXTURE. Figure S8. Principal component analyses of the African and European cohorts of the 1000GP, along with 43 Native American genomes and the TANGL cohort. Figure S9. Principal component analyses of the African and European cohorts of the $1000 \mathrm{GP}$, along with 43 Native American genomes and the TANGL cohort colored according to their proportions of global ancestry. Figure S10. Correlation of the principal component 1 and 2 values and the global ancestry proportions. For the TANGL.AFR. EUR.NAT cohort. Figure S11. Principal component analyses of the TANGL cohort colored according to their proportions of global ancestry. Figure S12. Correlation of the principal component 1 and 2 values and the global ancestry proportions for the TANGL cohort using common variants (MAF >10\%). Figure S13. Correlation of the principal component 1 and 2 values and the global ancestry proportions for the TANGL cohort using common variants (MAF 5-10\%). Figure S14. Pipeline of the curation of disease-causing variants in the TANGL cohort. Figure S15. Variant filtering of disease-causing variants in the TANGL cohort. Figure S16. Pedigrees of the families with pathogenic variants in PSEN1 (NM_000021). Figure S17. Pairwise identity by Descent (IBD) segments in the chromosomes that harbor the PSEN1 NM_000021 c.791C>T (p. Pro264Leu) variant. Figure S18. 
Pairwise identity by Descent (IBD) segments in the chromosomes that harbor the PSEN1 NM_000021 c.428T>C (p.lle143Thr) variant. Figure S19. Pairwise identity by Descent (IBD) segments in the chromosomes that harbor the PSEN1 NM_000021 c.356C>T (p.Thr119lle) variant in Colombian individuals. Figure S20. Pairwise identity by Descent (IBD) segments carrying the PSEN1 NM_000021 c.356C>T (p.Thr119lle) variant in Colombian and Argentinian individuals. Figure S21. Pedigrees of the family with a pathogenic variant in PSEN2 (NM_000447). Figure S22. Depth and allele balance indicate a duplication including APP. Figure S23. Pedigrees of the families with pathogenic variants in MAPT (NM_005910). Figure S24. Pairwise identity by Descent (IBD) segments in the chromosomes that harbor the MAPT NM_005910 c.1189C>T (p.Pro397Ser) variant. Figure S25. Pairwise identity by Descent (IBD) segments in the chromosomes that harbor the MAPT NM_005910 c.1189C > T (p.Pro397Ser) variant from Colombian and Spanish families. Figure S26. Pedigrees of the families with pathogenic variants in TBK1 (NM_013254). Figure S27. Pairwise identity by Descent (IBD) segments in the chromosomes that harbor TBK1 NM_013254 c.1257_1258del (p.Val421Cfs) variant. Figure S28. Pedigree of the family with a pathogenic variant in TARDBP (NM_007375). Figure S29. Pedigree of the family with a pathogenic variant in GRN (NM_002087). Figure S30. Pairwise identity by Descent (IBD) segments in the chromosomes that harbor SQSTM1 NM_003900 c.1175C > T (p.Pro392Leu) variant in the TANGL cohort. Figure S31. Alignment of the haplotypes that harbor SQSTM1 NM_003900 c.1175C > T (p.Pro392Leu) variant in the TANGL and the 1000GP cohort. Figure S32. Pedigrees of the families with pathogenic variants in TUBA4A (NM_006000). Figure S33. Pairwise identity by Descent (IBD) segments in the chromosomes that harbor TUBA4A NM_006000 c.820C>G (p.Pro274Ala) variant. Figure S34. Pedigrees of the families with pathogenic variants in UBQLN2 (NM_0013444) identified by the present study. Figure S35. Histological characterization of ceroid neuronal lipofuscinosis-4B (CNL4B) and Pedigree of the family. Figure S36. Histological characterization of hereditary diffuse leukoencephalopathy with spheroids (HDLS). Bottom row and Pedigree of the family. Figure S37. Alignment of the haplotypes that carry Strictly Damaging and Protein Truncating Variants in TREM2 present in more than 1 individual. Figure S38. Alignment of the haplotypes that carry Strictly Damaging and Protein Truncating Variants in $A B C A 7$ present in more than 1 individual. Figure S39. Alignment of the haplotypes that carry Strictly Damaging and Protein Truncating Variants in SORL1 present in more than 1 individual. Figure S40. Alignment of the haplotypes that carry Strictly Damaging and Protein Truncating Variants in ADAM10 present in more than 1 individual. Figure S41. Maps of Colombia representing the place of origin of the families with disease causing variants.

Additional file 2: Table S1. Demographic information of the included cohorts and their respective sub-cohorts.

Additional file 3: Table S2. Demographic information of the probands from included cohorts and their respective sub-cohorts.

Additional file 4: Table S3. GenBank accession numbers for the genes reported in the present study.

Additional file 5: Table S4. Mitochondrial haplogroups of the probands.

Additional file 6: Table S5. Y chromosome haplogroups of the male probands.

Additional file 7:. Supplementary methods.

Additional file 8: Table S6. Pathogenic variants identified in disease causing genes with additional information of the carriers.

Additional file 9: Table S7. Phenotypic information of the carriers of pathogenic variants in disease causing genes.

Additional file 10: Table S8. Neuropsychological battery performance in MAPT c.1189C $>T$ (p.Pro397Ser) carriers vs non-carriers according to their age groups and clinical diagnosis.

Additional file 11: Table S9. Family history of dementia and or motor neuron disease from the 566 probands.

Additional file 12: Table S10. Additional information of the carriers of Protein Truncating Variants (PVT) and Strictly Damaging variants (SD) in risk conferring genes.
Additional file 13: Table S11. Homozygosity by descent (HBD) in carriers of disease causing and risk conferring variants.

\section{Acknowledgements}

We thank the individuals and the families who participated in this study. We also thank the Grupo de Neurociencias de Antioquia (GNA) staff who helped with the participant recruitment, evaluation and sample processing. In addition, we acknowledge the collaboration of Juan Fernando Calle, Mauricio De la Espriella, Dora Lilia Hernández, Laura Ramírez-Aguilar, Nora Tobón, Ligia Eugenia Uribe, and the Instituto Neurológico de Colombia who helped with patient referral for the present study. We also acknowledge Juan P. Mejía at the GNA for the brain tissue processing and imaging. Elkin García Cifuentes, Juan E. Vélez, Manuela Gómez Vega, and Alberto Jaramillo-Jiménez for the thorough review of the participants medical records; Gregory Cooper at HudsonAlpha for helpful discussions about CNV calling; Kevin Wojta and Giovanni Coppola at the University of California Los Angeles for C9ORF72 sequencing; Jae-Hoon Sul and Sarah Spendlove for their time and thoughtful discussions about quality control and haplotype phasing; Ada A. Madejska and Sarah Eger at the University of California Santa Barbara for helping with local ancestry plotting; and Maria del Pilar Ramirez at the History department of the University of California Santa Barbara for helping with the historical background.

\section{Authors' contributions}

J.A-U, D.A., J.N.C., F.L., and K.S.K. conceived and designed the project with some discussion from EMR. J.A-U, D.A., M.G., D.A., L.V., S.M., L.H., L.M., D.V., J.M.S., D.M., and F.L. performed the clinical evaluations of the participants in Colombia. H.E.L provided the sociodemographic data from the Colombian participants. L.M., A.S., and F.P. gathered the pedigree information of the participants and collected samples. G.P.G. was in charge of the DNA extraction. E.I.S., T.I, and R.A. performed the clinical evaluation and provided the DNA sample from the participant from Argentina. R.S-V. performed the clinical evaluation and provided the DNA sample from the participant from Spain. J.N.C and R.M.M. performed the genomic sequencing. J.A-U. and B.W.K. did the variant curation process. J.A-U and S.L. performed Sanger sequencing of pathogenic and likely pathogenic variants. J.N.C. performed the copy number variation study. J.A-U, B.W.K, R.S., and N.P. performed the population structure, ancestry and identity by descent analyses with the guidance of S.R.B. A.V-L, and C.L.W. III performed the neuropathology assessments. J.A-U, J.N.C., and K.S.K. wrote the manuscript. S.R.B., F.L., and K.S.K. supervised the data generation and analyses. All authors read and approved the final manuscript.

\section{Funding}

Genome sequencing was supported by funding from the HudsonAlpha Foundation Memory and Mobility program. This project was funded by the Tau Consortium (F.L., K.S.K.), Larry L. Hillblom Foundation grant 2018-A-0004-NET (K.S.K.), Banner Alzheimer's Foundation (F.L., E.M.R.), NOMIS Foundation (E.M.R), and the National Institutes of Health grants GG013379 (K.S.K), RF1 AG062479 (K.S.K.), R01 AG055444 (E.M.R, F.L.), and P30 AG019610 (E.M.R).

\section{Availability of data and materials}

The genetic data obtained from the TANGL cohort (Raw data and BAM and VCF files aligned to hg19) have been deposited in the Grupo de Neurociencias de Antioquia (GNA) genetic data repository, Institutional repository of the Universidad de Antioquia (doi:10.5062/F4N58JNW) [157]. The Institutional Review Board (IRB) of the Medical Research Institute at the School of Medicine Universidad de Antioquia has restricted the deposition of the TANGL dataset to an institutional repository within the University of Antioquia. The TANGL dataset can be accessed and used by qualified researchers in collaborative projects involving the GNA. The application form for data access can be downloaded from the DOI link and should be emailed to juliana.acosta@gna. org.co. Applications are evaluated by GNA Neurogenetics Data Access Committee and response if given within 15 calendar days from application reception date. Novel "disease causing" and "risk conferring" variants that were not present in dbSNP and/or ClinVar databases were submitted to the National Center for Biotechnology Information ClinVar database [56]; https://www. ncbi.nlm.nih.gov/clinvar/ (accession numbers SCV001751539, SCV001751540, SCV001751542, SCV001751543, SCV001751544, SCV001751545, SCV001751546, SCV001751547, SCV001751549). The code used for the data 
analyses and plotting can be found at: https://github.com/acostauribe/TANGL (doi:10.5281/zenodo.5809622) [81].

\section{Declarations}

\section{Ethics approval and consent to participate}

Written informed consent following the guidelines of the Code of Ethics of the World Medical Association, Helsinki Declaration and Belmont Report was obtained from all participants or their legally authorized proxies. For the Colombian participants, the project was approved and overseen by the Institutional Review Board (IRB) of the Medical Research Institute, School of Medicine, Universidad de Antioquia (IORG0010323, FWA00028864). The IRB from "Instituto de Investigaciones Neurológicas Raúl Carrea - FLENI" (IORG0002360, FWA00022436) and "Hospital Clinic de Barcelona" (IORG0000975, FWA00000738) approved the use of the samples from Argentina and Spain. This research project conformed to the principles of the Helsinki Declaration and Belmont report. The brain donation and neuropathologic assessment were done with written informed consent and approved by the IRB of the Medical Research Institute, School of Medicine, Universidad de Antioquia.

\section{Consent for publication}

Not applicable

\section{Competing interests}

FL and EMR are the principal investigator of Alzheimer's prevention trials supported by NIH, philanthropy, and Genentech/Roche. EMR is also the principal investigator of Alzheimer's prevention trials supported by Eli Lilly, scientific advisor to Alzheon, Aural Analytics, Denali, Green Valley, Retromer Therapeutics \& Vaxxinity, a co-founder and share-holder of ALZPath, and co-inventor of a pending patent and inventor of existing patents related to Alzheimer's drug treatment discovery and the accelerated evaluation of Alzheimer's prevention therapies. The remaining authors declare that they have no competing interests.

\section{Author details}

${ }^{1}$ Neuroscience Research Institute and Department of Molecular Cellular and Developmental Biology, University of California, Santa Barbara, CA, USA. ${ }^{2}$ Grupo de Neurociencias de Antioquia, School of Medicine, Universidad de Antioquia, Medellín, Colombia. ${ }^{3}$ HudsonAlpha Institute for Biotechnology, Huntsville, AL, USA. ${ }^{4}$ Instituto Neurológico de Colombia (INDEC), Medellín, Colombia. ${ }^{5}$ Department of Internal Medicine, School of Medicine, Universidad del Sinú, Montería, Colombia. ${ }^{6}$ Department of Neurology, School of Medicine, Universidad de Antioquia, Medellín, Colombia. ${ }^{7}$ Banner Alzheimer's Institute, Phoenix, AZ, USA. ${ }^{8}$ Laboratorio de Enfermedades Neurodegenerativas (FleniCONICET), Buenos Aires, Argentina. ${ }^{9}$ Centro de Memoria y Envejecimiento (Fleni-CONICET), Buenos Aires, Argentina. ${ }^{10}$ Alzheimer's Disease and Other Cognitive Disorders Unit, Hospital Clínic de Barcelona, IDIBAPS and University of Barcelona, Barcelona, Spain. ${ }^{11}$ Neuropathology Section, Department of Pathology, University of Texas Southwestern Medical Center, Dallas, TX, USA. ${ }^{12}$ Instituto de Envejecimiento, Department of Psychiatry, School of Medicine, Pontifical Xaverian University, Bogotá, Colombia. ${ }^{13}$ Department of Mental Health, Hospital Universitario Santa Fe de Bogotá, Bogotá, Colombia. ${ }^{14}$ Department of Biostatistics, University of Washington, Seattle, WA, USA

\section{Received: 31 July 2021 Accepted: 26 February 2022}

Published online: 08 March 2022

\section{References}

1. Browning SR, Browning BL, Daviglus ML, Durazo-Arvizu RA, Schneiderman N, Kaplan RC, et al. Ancestry-specific recent effective population size in the Americas. Lohmueller KE, editor. PLoS Genet. 2018;14:e1007385. https://doi.org/10.1371/journal.pgen.1007385 [cited 2019 Mar 29]. Public Library of Science.

2. Ossa H, Aquino J, Pereira R, Ibarra A, Ossa RH, Pérez LA, et al. Outlining the ancestry landscape of colombian admixed populations. Chiang T-Y, editor. PLOSONE. 2016;11:e0164414 Public Library of Science; [cited 2018 Mar 17]. Available from: http://www.ncbi.nlm.nih.gov/pubmed/ 27736937.
3. Martin AR, Gignoux CR, Walters RK, Wojcik GL, Neale BM, Gravel S, et al. Human demographic history impacts genetic risk prediction across diverse populations. Am J Hum Genet. 2017;100:635-49 Cell Press. [cited 2018 Dec 9]. Available from: https://www.sciencedirect.com/ science/article/pii/S0002929717301076.

4. Visscher PM, Wray NR, Zhang Q, Sklar P, McCarthy MI, Brown MA, Yang J. 10 Years of GWAS Discovery: Biology, Function, and Translation. Am J Hum Genet. 2017;101(1):5-22. https://doi.org/10.1016/j.ajhg.2017.06. 005.

5. Wojcik GL, Graff M, Nishimura KK, Tao R, Haessler J, Gignoux CR, et al. Genetic analyses of diverse populations improves discovery for complex traits. Nature. 2019;570:514-8. https://doi.org/10.1038/s41586-0191310-4 [cited 2020 Feb 13].

6. Gravel S, Henn BM, Gutenkunst RN, Indap AR, Marth GT, Clark AG, et al. Demographic history and rare allele sharing among human populations. Proc Natl Acad Sci U S A. 2011;108:11983-8 [cited 2021 Apr 8]. Available from: www.pnas.org/cgi/doi/10.1073/pnas.1019276108. National Academy of Sciences.

7. Clark AG, Slatkin M, Ramos EM, Guttmacher AE, Mackay TFC, McCarthy $\mathrm{Ml}$, et al. Finding the missing heritability of complex diseases. Nature. 2009;461:747-53 [cited 2018 Nov 6]. Available from: http://www.nature. com/doifinder/10.1038/nature08494.

8. Cruts M. Alzheimer Disease \& Frontotemporal Dementia Mutation Database. [cited 2018 Jul 19]. Available from: http://www.molgen.vib-ua.be/ ADMutations

9. Ardila A, Lopera F, Rosselli M, Moreno S, Madrigal L, Arango-Lasprilla JC, et al. Neuropsychological profile of a large kindred with familial Alzheimer's disease caused by the E280A single presenilin-1 mutation. Arch Clin Neuropsychol. 2000;15:515-28 [cited 2018 Mar 14]. Available from: https://www.sciencedirect.com/science/article/pii/S088761779 9000414.

10. Aguirre-Acevedo DC, Gómez RD, Moreno S, Henao-Arboleda E, Motta M, Muñoz C, et al. Validez y fiabilidad de la batería neuropsicológica CERAD-Col. Rev Neurol. 2007;45:655-60.

11. McKhann GM, Knopman DS, Chertkow H, Hyman BT, Jack CR, Kawas $\mathrm{CH}$, et al. The diagnosis of dementia due to Alzheimer's disease: recommendations from the National Institute on Aging-Alzheimer's association workgroups on diagnostic guidelines for Alzheimer's disease. Alzheimers Dement. 2011;7:263-9 [cited 2019 Mar 27]. Available from: https://www.sciencedirect.com/science/article/pii/S15525260110010 14?via\%3Dihub.

12. Gorno-Tempini ML, Hillis AE, Weintraub S, Kertesz A, Mendez M, Cappa SF, et al. Classification of primary progressive aphasia and its variants. Neurology. 2011;76:1006-14 [cited 2019 Mar 27]. Available from: http:// www.ncbi.nlm.nih.gov/pubmed/21325651.

13. Rascovsky K, Hodges JR, Knopman D, Mendez MF, Kramer JH, Neuhaus J, et al. Sensitivity of revised diagnostic criteria for the behavioural variant of frontotemporal dementia. Brain Narnia. 2011;134:2456-77 [cited 2019 Mar 27]. Available from: https://academic.oup.com/brain/artic le-lookup/doi/10.1093/brain/awr179.

14. Höglinger GU, Respondek G, Stamelou M, Kurz C, Josephs KA, Lang AE, et al. Clinical diagnosis of progressive supranuclear palsy: the movement disorder society criteria. Mov Disord. 2017;32:853-64[cited 2019 Mar 27]. Available from. https://doi.org/10.1002/mds.26987.

15. Strong MJ, Abrahams S, Goldstein LH, Woolley S, Mclaughlin P, Snowden J, et al. Amyotrophic lateral sclerosis - frontotemporal spectrum disorder (ALS-FTSD): revised diagnostic criteria. Amyotroph Lateral Scler Frontotemporal Degener. 2017;18:153-74 [cited 2021 Jul 20]. Available from: http://www.ncbi.nlm.nih.gov/pubmed/28054827.

16. Li H, Durbin R. Fast and accurate short read alignment with burrowswheeler transform. Bioinformatics. 2009;25:1754-60 [cited 2018 Mar 14]. Available from: http://www.ncbi.nlm.nih.gov/pubmed/19451168.

17. Tarasov A, Vilella AJ, Cuppen E, Nijman IJ, Prins P. Sambamba: fast processing of NGS alignment formats. Bioinformatics. 2015:31:2032-4 [cited 2019 Oct 29]. Available from: http://www.ncbi.n/m.nih.gov/ pubmed/25697820.

18. McKenna A, Hanna M, Banks E, Sivachenko A, Cibulskis K, Kernytsky A, et al. The genome analysis toolkit: a MapReduce framework for analyzing next-generation DNA sequencing data. Genome Res. 2010;20:1297-303 [cited 2021 Aug 7]. Available from: https://genome. cshlp.org/content/20/9/1297.full. 
19. Zhao S, Agafonov O, Azab A, Stokowy T, Hovig E. Accuracy and efficiency of germline variant calling pipelines for human genome data. Sci Rep. 2020;10 [cited 2021 Oct 2]. Available from: https://pubmed. ncbi.nlm.nih.gov/33214604/.

20. Cingolani P, Platts A, Wang LLL, Coon M, Nguyen T, Wang LLL, et al. A program for annotating and predicting the effects of single nucleotide polymorphisms, SnpEff: SNPs in the genome of Drosophila melanogaster strain w1118; iso-2; iso-3. Fly (Austin). 2012;6:80-92.

21. Tan A, Abecasis GR, Kang HM. Unified representation of genetic variants. Bioinformatics. 2015;31:2202-4 [cited 2020 Jan 29]. Available from: https://www.ncbi.nlm.nih.gov/pubmed/25701572.

22. Howe KL, Achuthan P, Allen J, Allen J, Alvarez-Jarreta J, Ridwan Amode M, et al. Ensembl 2021. Nucleic Acids Res. 2021;49:D884-91 [cited 2021 Dec 13]. Available from: https://academic.oup.com/nar/article/49/D1/ D884/5952199.

23. Kircher M, Witten DM, Jain P, O'roak BJ, Cooper GM, Shendure J, et al. A general framework for estimating the relative pathogenicity of human genetic variants. Nat Genet. 2014;46:310-5 [cited 2018 Dec 12]. Available from: http://www.nature.com/articles/ng.2892.

24. Altshuler DM, Durbin RM, Abecasis GR, Bentley DR, Chakravarti A, Clark AG, et al. An integrated map of genetic variation from 1,092 human genomes. Nature. 2012;491:56-65 [cited 2019 Jul 29]. Available from: http://www.nature.com/articles/nature11632.

25. NIH NHLBI, National Heart, Lung and BI. NHLBI Trans-Omics for Precision Medicine WGS-About TOPMed. [cited 2020 Jan 29]. Available from: https://www.nhlbiwgs.org/

26. Zhao H, Sun Z, Wang J, Huang H, Kocher J-PP, Wang L. CrossMap: a versatile tool for coordinate conversion between genome assemblies. Bioinformatics. 2013;30:1006-7.

27. Liu X, White S, Peng B, Johnson AD, Brody JA, Li AH, et al. WGSA: an annotation pipeline for human genome sequencing studies. J Med Genet. 2015;53:111-2 [cited 2020 Jan 29]. Available from: http://www. pubmedcentral.nih.gov/articlerender.fcgi?artid=PMC5124490.

28. Lek M, Karczewski KJ, Minikel EV, Samocha KE, Banks E, Fennell T, et al. Analysis of protein-coding genetic variation in 60,706 humans. Nature. 2016:536:285-91 [cited 2018 Mar 20]. Available from: http://www. nature.com/articles/nature19057.

29. Karczewski KJ, Francioli LC, Tiao G, Cummings BB, Alföldi J, Wang Q, et al. Variation across 141,456 human exomes and genomes reveals the spectrum of loss-of-function intolerance across human protein-coding genes. bioRxiv. 2019;531210 [cited 2020 Jan 29]. Available from: https:// www.biorxiv.org/content/10.1101/531210v3.

30. Exome Variant Server; NHLBI GO Exome Sequencing Project (ESP). Seattle; Available from: http://evs.gs.washington.edu/EVS

31. Walter K, Min JL, Huang J, Crooks L, Memari Y, McCarthy S, et al. The UK10K project identifies rare variants in health and disease. Nature. 2015;526:82-9 [cited 2021 Dec 13]. Available from:/pmc/articles/ PMC4773891/.

32. Sherry ST, Ward MH, Kholodov M, Baker J, Phan L, Smigielski EM, et al. dbSNP: the NCBI database of genetic variation. Nucleic Acids Res. 2001;29:308-11 [cited 2020 Jan 29]. Available from: http://www.ncbi. nlm.nih.gov/pubmed/11125122.

33. Danecek P, Auton A Abecasis G, Albers CA, Banks E, DePristo MA et al. The variant call format and VCFtools. Bioinformatics. 2011;27:2156-8.

34. Manichaikul A, Mychaleckyj JC, Rich SS, Daly K, Sale M, Chen W-M. Robust relationship inference in genome-wide association studies. Bioinformatics. 2010:26:2867-73 [cited 2019 Oct 29]. Available from: http://www.ncbi.nlm.nih.gov/pubmed/20926424.

35. Purcell S, Neale B, Todd-Brown K, Thomas L, Ferreira MAR, Bender D, et al. PLINK: a tool set for whole-genome association and populationbased linkage analyses. Am J Hum Genet. 2007;81:559-75 [cited 2018 Mar 14]. Available from: https://www.sciencedirect.com/science/artic le/pii/S0002929707613524.

36. Chang CC, Chow CC, Tellier LCAM, Vattikuti S, Purcell SM, Lee JJ. Secondgeneration PLINK: Rising to the challenge of larger and richer datasets. Gigascience. 2015;4:7. https://doi.org/10.1186/s13742-015-0047-8,

37. Anderson CA, Pettersson FH, Clarke GM, Cardon LR, Morris AP, Zondervan KT. Data quality control in genetic case-control association studies. Nat Protoc. 2010;5:1564-73 [cited 2019 Oct 29]. Available from: https:// www.nature.com/articles/nprot.2010.116.
38. Arango D, Cruts M, Torres O, Backhovens H, Serrano ML, Villareal E, et al. Systematic genetic study of Alzheimer disease in Latin America: mutation frequencies of the amyloid $\beta$ precursor protein and presenilin genes in Colombia. Am J Med Genet. 2001;103:138-43 [cited 2018 Mar 17]. Available from: https://doi.org/10.1002/1096-8628(20011001)103: 2<138::AID-AJMG1529>3.0.CO;2-8.

39. Itzcovich T, Chrem-Méndez P, Vázquez S, Barbieri-Kennedy M, Niikado

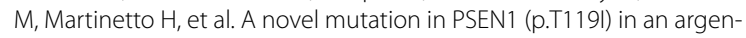
tine family with early- and late-onset Alzheimer's disease. Neurobiol Aging. 2020;85:155.e9-155.e12.

40. Das S, Forer L, Schönherr S, Sidore C, Locke AE, Kwong A, et al. Nextgeneration genotype imputation service and methods. Nat Genet. 2016;48:1284-7 [cited 2021 Dec 13]. Available from: https://www. nature.com/articles/ng.3656.

41. Rayner W. HRC or 1000 G Imputation preparation and checking. Available from: https://www.well.ox.ac.uk/ wrayner/tools/\#Checking

42. Borrego-Écija S, Antonell A, Puig-Butillé JA, Pericot I, Prat-Bravo C, Abellan-Vidal MT, et al. Novel P397S MAPT variant associated with late onset and slow progressive frontotemporal dementia. Ann Clin Transl Neurol. 2019:6:1559-65.

43. Sentieon Genomics software. Available from: https://support.sentieon. com

44. Moreno-Estrada A, Gravel S, Zakharia F, McCauley JL, Byrnes JK, Gignoux $\mathrm{CR}$, et al. Reconstructing the population genetic history of the Caribbean. Tarazona-Santos E, editor. PLoS Genet. 2013:9:e1003925[cited 2019 Aug 7]. Available from:. https://doi.org/10.1371/journal.pgen. 1003925.

45. Mao X, Bigham AW, Mei R, Gutierrez G, Weiss KM, Brutsaert TD, et al. A genomewide admixture mapping panel for Hispanic/Latino populations. Am J Hum Genet. 2007;80:1171-8.

46. Alexander DH, Novembre J, Lange K. Fast model-based estimation of ancestry in unrelated individuals. Genome Res. 2009;19:1655-64 [cited 2019 Sep 2]. Available from: http://www.ncbi.nlm.nih.gov/pubmed/ 19648217.

47. R Core Team. R: a language and environment for statistical computing. Available from: https://www.r-project.org/

48. Weissensteiner $\mathrm{H}$, Pacher D, Kloss-Brandstätter A, Forer L, Specht G, Bandelt HJ, et al. HaploGrep 2: mitochondrial haplogroup classification in the era of high-throughput sequencing. Nucleic Acids Res. 2016;44:W58-63 [cited 2020 Oct 7]. Available from: http://haplogrep. uibk.ac.at.

49. Poznik GD. Identifying Y-chromosome haplogroups in arbitrarily large samples of sequenced or genotyped men. bioRxiv. 2016;088716. [cited 2020 Oct 7]. Available from. https://doi.org/10.1101/088716.

50. Delaneau O, Marchini J, Zagury JF. A linear complexity phasing method for thousands of genomes. Nat Methods. 2012;9:179-81.

51. O'Connell J, Gurdasani D, Delaneau O, Pirastu N, Ulivi S, Cocca M, et al. A general approach for haplotype phasing across the full spectrum of relatedness. Gibson G, editor. PLoS Genet. 2014;10:e1004234. https:// doi.org/10.1371/journal.pgen.1004234 [cited 2019 Oct 30].

52. Maples BK, Gravel S, Kenny EE, Bustamante CD. RFMix: a discriminative modeling approach for rapid and robust local-ancestry inference. Am J Hum Genet. 2013:93:278-88 Available from: https://www.sciencedirect. com/science/article/pii/S0002929713002899\#fig3. [cited 2019 Sep 2].

53. Price AL, Patterson NJ, Plenge RM, Weinblatt ME, Shadick NA, Reich D. Principal components analysis corrects for stratification in genomewide association studies. Nat Genet. 2006;38:904-9.

54. Novembre J, Williams R, Hossein P, Wang Y. PCAviz: visualizing principal components analysis. R package version 0.3-37. 2019. Available from: http://github.com/NovembreLab/PCAviz

55. Richards S, Aziz N, Bale S, Bick D, Das S, Gastier-Foster J, et al. Standards and guidelines for the interpretation of sequence variants: a joint consensus recommendation of the American College of Medical Genetics and Genomics and the Association for Molecular Pathology. Genet Med. 2015;17:405-24 Available from: http://www.nature.com/articles/ gim201530. [cited 2018 Mar 20].

56. Landrum MJ, Lee JM, Benson M, Brown GR, Chao C, Chitipiralla S, et al. ClinVar: improving access to variant interpretations and supporting evidence. Nucleic Acids Res. 2018;46:D1062-7 [cited 2022 Jan 21]. Available from: https://pubmed.ncbi.nlm.nih.gov/29165669/. 
57. Allot A, Peng Y, Wei C-HH, Lee K, Phan L, Lu Z. LitVar: a semantic search engine for linking genomic variant data in PubMed and PMC. Nucleic Acids Res. 2018;46:W530-6 [cited 2020 Jun 3]. Available from: https:// pubmed.ncbi.nlm.nih.gov/29762787/.

58. Guerreiro RJ, Baquero M, Blesa R, Boada M, Brás JM, Bullido MJ, et al. Genetic screening of Alzheimer's disease genes in Iberian and African samples yields novel mutations in presenilins and APP. Neurobiol Aging 2010;31:725-31 [cited 2018 Mar 16]. Available from: https://www.scien cedirect.com/science/article/pii/S0197458008002194\#fig2.

59. DeJesus-Hernandez M, Mackenzie IR, Boeve BF, Boxer AL, Baker M, Rutherford NJ, et al. Expanded GGGGCC hexanucleotide repeat in noncoding region of C9ORF72 causes chromosome 9p-linked FTD and ALS. Neuron. 2011;72:245-56.

60. Dolzhenko E, van Vugt JJFA, Shaw RJ, Bekritsky MA, Van Blitterswijk $M$, Narzisi $G$, et al. Detection of long repeat expansions from PCR-free whole-genome sequence data. Genome Res. 2017;27:1895-903 [cited 2021 Sep 20]. Available from: https://pubmed.ncbi.nlm.nih.gov/28887 402/.

61. Rausch T, Zichner T, Schlattl A, Stutz AM, Benes V, Korbel JO. DELLY: structural variant discovery by integrated paired-end and split-read analysis. Bioinformatics. 2012;28:i333-9 [cited 2020 Feb 19]. Available from: https://academic.oup.com/bioinformatics/article-lookup/doi/10. 1093/bioinformatics/bts378

62. Zhu M, Need AC, Han Y, Ge D, Maia JM, Zhu Q, et al. Using ERDS to infer copy-number variants in high-coverage genomes. Am J Hum Genet. 2012;91:408-21.

63. Abyzov A, Urban AE, Snyder M, Gerstein M. CNVnator: an approach to discover, genotype, and characterize typical and atypical CNVs from family and population genome sequencing. Genome Res. 2011;21:974-84

64. Xi R, Lee S, Xia Y, Kim TM, Park PJ. Copy number analysis of wholegenome data using BIC-seq2 and its application to detection of cancer susceptibility variants. Nucleic Acids Res. 2016;44:6274-86.

65. Robinson JT, Thorvaldsdóttir H, Winckler W, Guttman M, Lander ES, Getz G, et al. Integrative genomics viewer. Nat Biotechnol 29, 24-26 (2011). https://doi.org/10.1038/nbt.1754

66. Hiatt SM, Lawlor JMJ, Handley LH, Ramaker RC, Rogers BB, Partridge $\mathrm{EC}$, et al. Long-read genome sequencing for the molecular diagnosis of neurodevelopmental disorders. Hum Genet Genomics Adv. 2021;2:100023 [cited 2021 Sep 20]. Available from: https://pubmed. ncbi.nlm.nih.gov/33937879/.

67. Mirra SS, Heyman A, McKeel D, Sumi SM, Crain BJ, Brownlee LM, et al. The consortium to establish a registry for Alzheimer's disease (CERAD). Part II. Standardization of the neuropathologic assessment of Alzheimer's disease. Neurology. 1991;41:479-86 [cited 2021 Dec 27]. Available from: https://pubmed.ncbi.nlm.nih.gov/2011243/.

68. Vonsattel JPG, del Amaya MP, Keller CE. Twenty-first century brain banking. Processing brains for research: the Columbia University methods. Acta Neuropathol. 2008;115:509-32 [cited 2021 Dec 27]. Available from: https://link.springer.com/article/10.1007/s00401-007-0311-9.

69. Bellenguez C, Charbonnier C, Grenier-Boley B, Quenez O, Le Guennec K, Nicolas $\mathrm{G}$, et al. Contribution to Alzheimer's disease risk of rare variants in TREM2, SORL1, and ABCA7 in 1779 cases and 1273 controls. Neurobiol Aging. 2017;59:220.e1-220.e9 [cited 2018 Mar 16]. Available from: https://ac.els-cdn.com/S0197458017302324/1-s2.0-S01974580173023 24-main.pdf?_tid=40b88b1a-3e1c-4262-b7cb-33ec8ae2bcfa\&acdnat= 1521235271_f9c9f72160c28f3f72f5a3e1e0ed5885.

70. Zhou SL, Tan CC, Hou XH, Cao XP, Tan L, Yu JT. TREM2 variants and neurodegenerative diseases: a systematic review and meta-analysis. J Alzheimer's Dis. 2019;68(3):1171-84.https://doi.org/10.3233/JAD-181038.

71. Steinberg S, Stefansson $H$, Jonsson $T$, Johannsdottir H, Ingason A, Helgason $\mathrm{H}$, et al. Loss-of-function variants in ABCA7 confer risk of Alzheimer's disease. Nat Genet. 2015;47:445-7 [cited 2018 Mar 16]. Available from: http://www.nature.com/.

72. Cuyvers E, De Roeck A, Van den Bossche T, Van Cauwenberghe C, Bettens K, Vermeulen S, et al. Mutations in ABCA7 in a Belgian cohort of Alzheimer's disease patients: a targeted resequencing study. Lancet Neurol. 2015;14:814-22. https://doi.org/10.1016/S1474-4422(15)001337. [cited 2018 Sep 12].

73. Campion D, Charbonnier C, Nicolas G. SORL1 genetic variants and Alzheimer disease risk: a literature review and meta-analysis of sequencing data. Acta Neuropathol. 2019:173-86. https://doi.org/10.1007/s00401019-01991-4 [cited 2020 Sep 16].

74. Ng PC, Henikoff S. Predicting deleterious amino acid substitutions. Genome Res. 2001;11:863-74 [cited 2020 Oct 7]. Available from: https:// pubmed.ncbi.nlm.nih.gov/11337480/.

75. Adzhubei IA, Schmidt S, Peshkin L, Ramensky VE, Gerasimova A, Bork P, et al. A method and server for predicting damaging missense mutations. Nat Methods. 2010:248-9 [cited 2020 Oct 7]. Available from: https://pubmed.ncbi.nlm.nih.gov/20354512/.

76. Schwarz JM, Cooper DN, Schuelke M, Seelow D. Mutationtaster2: mutation prediction for the deep-sequencing age. Nat Methods. 2014:361-2 [cited 2020 Oct 7]. Available from https://doi.org/10.1038/nmeth.2890.

77. Kim M, Suh J, Romano D, Truong MH, Mullin K, Hooli B, et al. Potential late-onset Alzheimer's disease-associated mutations in the ADAM10 gene attenuate a-secretase activity. Hum Mol Genet. 2009;18:3987-96 [cited 2020 Sep 14]. Available from: https://academic.oup.com/hmg/ article/18/20/3987/645530.

78. Suh J, Choi SH, Romano DM, Gannon MA, Lesinski AN, Kim DY, et al. ADAM10 missense mutations potentiate $\beta$-amyloid accumulation by impairing prodomain chaperone function. Neuron. 2013;80:385-401.

79. den Dunnen JT, Dalgleish R, Maglott DR, Hart RK, Greenblatt MS, Mcgowan-Jordan J, et al. HGVS recommendations for the description of sequence variants: 2016 update. Hum Mutat. 2016;37:564-9 [cited 2018 Mar 20]. Available from: http://doi.wiley.com/10.1002/humu.22981.

80. Zhou Y, Browning SR, Browning BL. A fast and simple method for detecting identity-by-descent segments in large-scale data. Am J Hum Genet. 2020;106:426-37.

81. Acosta-Uribe J, Singhal R. TANGL: set of scripts used for the paper "a neurodegenerative disease landscape of rare mutations in Colombia due to founder effects". Github. 2021; Available from: https://github. com/acostauribe/TANGL.

82. Auton A, Abecasis GR, Altshuler DM, Durbin RM, Bentley DR, Chakravarti $A$, et al. A global reference for human genetic variation. Nature. 2015;526:68-74. https://doi.org/10.1038/nature15393.

83. Rishishwar L, Conley $A B$, Wigington $C H$, Wang L, Valderrama-Aguirre $A$, Jordan IK, et al. Ancestry, admixture and fitness in Colombian genomes. Sci Rep. 2015;5:12376 [cited 2018 Mar 26]. Available from: http://www. nature.com/articles/srep12376.

84. Carvajal-Carmona LG, Soto ID, Pineda N, Ortíz-Barrientos D, Duque C, Ospina-Duque J, et al. Strong Amerind/white sex bias and a possible Sephardic contribution among the founders of a population in Northwest Colombia. Am J Hum Genet. 2000;67:1287-95 [cited 2018 Apr 11]. Available from: http://www.ncbi.nlm.nih.gov/pubmed/11032790.

85. Mathias RA, Taub MA, Gignoux CR, Fu W, Musharoff S, O'Connor TD, et al. A continuum of admixture in the Western hemisphere revealed by the African diaspora genome. Nat Commun. 2016;7:12522 [cited 2019 Mar 29]. Available from: http://www.nature.com/articles/ncomms 12522

86. Lopera F, Ardilla A, Martinez A, Madrigal L, Arango-Viana J, Lemere C, et al. Clinical features of early-onset Alzheimer disease in a large kindred with an E280A presenilis-1 mutation. Am J Ophthalmol. 1997;124:137-8 [cited 2020 Mar 4]. Available from: https://linkinghub.elsevier.com/retri eve/pii/S0002939414716770.

87. Ramirez Aguilar L, Acosta-Uribe J, Giraldo MM, Moreno S, Baena A, Alzate D, et al. Genetic origin of a large family with a novel PSEN1 mutation (Ile416Thr). Alzheimers Dement. 2019;15:709-19 [cited 2021 Jul 20]. Available from: https://alz-journals.onlinelibrary.wiley.com/doi/10. 1016/j.jalz.2018.12.010.

88. Gómez-Tortosa E, Barquero S, Barón M, Gil-Neciga E, Castellanos F, Zurdo M, et al. Clinical-genetic correlations in familial Alzheimer's disease caused by presenilin 1 mutations. J Alzheimers Dis. 2010;19:87384 [cited 2018 Jul 24]. Available from: http://www.ncbi.nlm.nih.gov/ pubmed/20157243.

89. Campion D, Flaman JM, Brice A, Hannequin D, Dubois B, Martin C, et al. Mutations of the presenilin I gene in families with early-onset alzheimer's disease. Hum Mol Genet. 1995;4:2373-7 [cited 2020 Oct 19]. Available from: https://pubmed.ncbi.nlm.nih.gov/8634712/.

90. Mutations Database, Alzforum. [cited 2020 Sep 1]. Available from: http://www.alzforum.org/mutations

91. Rogaeva EA, Fafel KC, Song YQ, Medeiros H, Sato C, Liang Y, et al. Screening for PS1 mutations in a referral-based series of AD cases: 21 novel mutations. Neurology. 2001;57:621-5. 
92. Miravalle L, Calero M, Takao M, Roher AE, Ghetti B, Vidal R. Amino-terminally truncated $A \beta$ peptide species are the main component of cotton wool plaques t. Biochemistry. 2005;44:10810-21 [cited 2021 Mar 11]. Available from: https://pubmed.ncbi.nlm.nih.gov/16086583/.

93. Jiménez Caballero PE, De Diego BC, Martin Correa E, Serviá Candela M, Marsal AC. A novel presenilin 1 mutation (V261L) associated with presenile Alzheimer's disease and spastic paraparesis. Eur J Neurol. 2008;15:991-4 [cited 2021 Mar 11]. Available from: https://pubmed. ncbi.nlm.nih.gov/18637955/.

94. Tabira T, De Chui H, Nakayama H, Kuroda S, Shibuya M. Alzheimer's disease with spastic paresis and cotton wool type plaques. J Neurosci Res. 2002:367-72 [cited 2018 Sep 7]. Available from: http://doi.wiley.com/10. 1002/jnr.10392.

95. O'Riordan S, McMonagle P, Janssen JC, Fox NC, Farrell M, Collinge J, et al. Presenilin-1 mutation (E280G), spastic paraparesis, and cranial MRI white-matter abnormalities. Neurology. 2002;59:1108-10 [cited 2020 Oct 9]. Available from: https://pubmed.ncbi.nlm.nih.gov/12370477/.

96. Campion D, Dumanchin C, Hannequin D, Dubois B, Belliard S, Puel M, et al. Early-onset autosomal dominant Alzheimer disease: prevalence, genetic heterogeneity, and mutation spectrum. Am J Hum Genet. 1999;65:664-70.

97. Raux G, Guyant-Maréchal L, Martin C, Bou J, Penet C, Brice A, et al. Molecular diagnosis of autosomal dominant early onset Alzheimer's disease: an update. J Med Genet. 2005:793-5 [cited 2021 Sep 20]. Available from: https://pubmed.ncbi.nlm.nih.gov/16033913/.

98. Jacquemont ML, Campion D, Hahn V, Tallaksen C, Frebourg T, Brice A, et al. Spastic paraparesis and atypical dementia caused by PSEN1 mutation (P264L), responsible for Alzheimer's disease. J Med Genet. 2002;39 [cited 2021 Sep 20]. Available from: https://pubmed.ncbi.nlm.nih.gov/ $11836371 /$.

99. Dumanchin C, Tournier I, Martin C, Didic M, Belliard S, Carlander B, et al. Biological effects of four PSEN1 gene mutations causing Alzheimer disease with spastic paraparesis and cotton wool plaques. Hum Mutat. 2006;27:1063 [cited 2020 Jan 16]. Available from: http://doi.wiley.com/ 10.1002/humu.9458.

100. Poorkaj P, Sharma V, Anderson L, Nemens E, Alonso ME, Orr H, et al. Missense mutations in the chromosome 14 familial Alzheimer's disease presenilin 1 gene. Hum Mutat. 1998;11:216-21.

101. Mahoney CJ, Downey LE, Beck J, Liang Y, Mead S, Perry RJ, et al. The presenilin 1 P264L mutation presenting as non-fluent/agrammatic primary progressive aphasia. J Alzheimer's dis. J Alzheimers Dis. 2013;36:239-43 [cited 2021 Sep 20]. Available from: https://pubmed.ncbi.nlm.nih.gov/ 23579325/.

102. Lohmann E, Guerreiro RJ, Erginel-Unaltuna N, Gurunlian N, Bilgic B, Gurvit $\mathrm{H}$, et al. Identification of PSEN1 and PSEN2 gene mutations and variants in Turkish dementia patients. Neurobiol Aging. 2012;33:1850. e17-27.

103. Ishizuka T, Nakamura M, Ichiba M, Fujita S, Takeuchi K, Fujimoto T, et al. Different clinical phenotypes in siblings with a Presenilin-1 P264L mutation. Dement Geriatr Cogn Disord. 2012;33:132-40.

104. Lanoiselée H-MM, Nicolas G, Wallon D, Rovelet-Lecrux A, Lacour M, Rousseau S, et al. APP, PSEN1, and PSEN2 mutations in early-onset Alzheimer disease: a genetic screening study of familial and sporadic cases. Miller BL, editor. PLoS Med. 2017;14:e1002270[cited 2018 Mar 27]. Available from. https://doi.org/10.1371/journal.pmed.1002270.

105. Koriath C, Kenny J, Adamson G, Druyeh R, Taylor W, Beck J, et al. Predictors for a dementia gene mutation based on gene-panel next-generation sequencing of a large dementia referral series. Mol Psychiatry. 2020;25:3399-412 [cited 2021 Mar 11]. Available from: https://www. nature.com/articles/s41380-018-0224-0.

106. Hippen AA, Ebbert MTW, Norton MC, Tschanz JAT, Munger RG, Corcoran CD, et al. Presenilin E318G variant and Alzheimer's disease risk: the Cache County study. BMC Genomics; 2016;17:295-299. [cited 2019 May 29]. Available from: https://www.com-mendeley-prod-publicshar ing-pdfstore.s3.eu-west-1.amazonaws.com/9478-PUBMED/10.1186/ s12864-016-2786-z/12864_2016_Article_2786_pdf.pdf?X-Amz-Secur ity-Token=FQoGZXIvYXdzEFoaDJvrD6JHLPgKR7VrOiKfBG\%2BjE4yKjxx CgbRpXMtyOFbFXxWMP3WOIXuBX9KmcHPxteQzaB

107. Perrone F, Bjerke M, Hens E, Sieben A, Timmers M, De Roeck A, et al. Amyloid- $\beta 1$-43cerebrospinal fluid levels and the interpretation of APP, PSEN1 and PSEN2 mutations. Alzheimers Res Ther. 2020;12:1-14[cited 2021 May 16]. Available from:. https://doi.org/10. 1186/s13195-020-00676-5.

108. Gao Y, Ren RJ, Zhong ZL, Dammer E, Zhao QH, Shan S, et al. Mutation profile of APP, PSEN1, and PSEN2 in Chinese familial Alzheimer's disease. Neurobiol Aging. 2019;77:154-7 [cited 2020 Oct 19]. Available from: https://linkinghub.elsevier.com/retrieve/pii/S0197458019300326.

109. Freischmidt A, Wieland T, Richter B, RufW, Schaeffer V, Müller K, et al. Haploinsufficiency of TBK1 causes familial ALS and fronto-temporal dementia. Nat Neurosci. 2015;18:631-6 [cited 2020 Jun 18]. Available from: http://www.nature.com/articles/nn.4000.

110. Bonnard M. Deficiency of T2K leads to apoptotic liver degeneration and impaired NF-kappaB-dependent gene transcription. EMBO J. 2000;19:4976-85 [cited 2021 Jan 10]. Available from: https://www. embopress.org/doi/full/10.1093/emboj/19.18.4976.

111. Rutherford NJ, Zhang YJ, Baker M, Gass JM, NCA F, Xu YF, et al. Novel mutations in TARDBP(TDP-43) in patients with familial amyotrophic lateral sclerosis. Cox GA, editor. PLoS Genet. 2008;4:e1000193. https:// doi.org/10.1371/journal.pgen.1000193 [cited 2019 Jan 28].

112. Corrado L, Ratti A, Gellera C, Buratti E, Castellotti B, Carlomagno Y, et al. High frequency of TARDBP gene mutations in Italian patients with amyotrophic lateral sclerosis. Hum Mutat. 2009;30:688-94 Available from: http://doi.wiley.com/10.1002/humu.20950.

113. Ramos EM, Koros C, Dokuru DR, Van Berlo V, Kroupis C, Wojta K, et al. Frontotemporal dementia spectrum: first genetic screen in a Greek cohort. Neurobiol Aging. 2019;75:224.e1-8 [cited 2019 Jan 29]. Available from: https://www-sciencedirect-com.proxy.library.ucsb.edu:9443/ science/article/pii/S0197458018303932?via\%3Dihub.

114. Le Ber I, Camuzat A, Guerreiro R, Bouya-Ahmed K, Bras J, Nicolas G, et al. SQSTM1 mutations in french patients with frontotemporal dementia or frontotemporal dementia with amyotrophic lateral sclerosis. JAMA Neurol. 2013;70:1403-10 [cited 2018 Aug 30]. Available from: http:// www.ncbi.nlm.nih.gov/pubmed/24042580.

115. Fecto F, Yan J, Vemula SP, Liu E, Yang Y, Chen W, et al. SQSTM1 mutations in familial and sporadic amyotrophic lateral sclerosis. Arch Neurol. 2011;68:1440-6 [cited 2018 Aug 28]. Available from: http://archneur. jamanetwork.com/article.aspx?doi=10.1001/archneurol.2011.250.

116. Laurin N, Brown JP, Morissette J, Raymond V. Recurrent mutation of the gene encoding sequestosome 1 (SQSTM1/p62) in Paget disease of bone. Am J Hum Genet. 2002;70:1582-8 [cited 2020 Dec 11]. Available from: http://www.cell.com/article/S0002929707607113/ fulltext.

117. Cuyvers E, van der Zee J, Bettens K, Engelborghs S, Vandenbulcke M, Robberecht C, et al. Genetic variability in SQSTM1 and risk of earlyonset Alzheimer dementia: a European early-onset dementia consortium study. Neurobiol Aging. 2015;36:2005.e15-22 [cited 2018 Aug 30]. Available from: https://com-mendeley-prod-publicsharing-pdfstore.s3. eu-west-1.amazonaws.com/0864-ELSEVIER/10.1016/j.neurobiolaging. 2015.02.014/Genetic_variability_in_SQSTM1_and_risk_of_early_onset Alzheimer_dementia_A_European_early_onset_dementia_conso rtium_study.pdf?X-

118. van der Zee J, Van Langenhove T, Kovacs GG, Dillen L, Deschamps W, Engelborghs S, et al. Rare mutations in SQSTM1 modify susceptibility to frontotemporal lobar degeneration. Acta Neuropathol. 2014;128:397410 [cited 2018 Aug 30]. Available from: http://link.springer.com/10. 1007/s00401-014-1298-7.

119. Chung PYJ, Beyens G, Guañabens N, Boonen S, Papapoulos S, Karperien $M$, et al. Founder effect in different european countries for the recurrent P392L SQSTM1 mutation in Paget's disease of bone. Calcif Tissue Int. 2008;83:34-42 [cited 2018 Aug 29]. Available from: http://link.springer. com/10.1007/s00223-008-9137-2.

120. Andersen PM, Sims KB, Xin WW, Kiely R, O’Neill G, Ravits J, et al. Sixteen novel mutations in the $\mathrm{cu} / \mathrm{Zn}$ superoxide dismutase gene in amyotrophic lateral sclerosis: a decade of discoveries, defects and disputes. Amyotroph Lateral Scler Other Mot Neuron Disord. 2003:62-73 [cited 2020 Dec 13]. Available from: https://www.tandfonline.com/action/ journallnformation?journalCode=iafd20.

121. Lattante S, Conte A, Zollino M, Luigetti M, Del Grande A, Marangi G, et al. Contribution of major amyotrophic lateral sclerosis genes to the etiology of sporadic disease. Neurology. 2012;79:66-72 [cited 2020 Dec 13]. Available from: http://www.neurology.org/cgi/doi/10.1212/WNL. Ob013e31825dceca. 
122. Kenna KP, McLaughlin RL, Byrne S, Elamin M, Heverin M, Kenny EM, et al. Delineating the genetic heterogeneity of ALS using targeted highthroughput sequencing. J Med Genet. 2013;50:776-83.

123. Lenk GM, Ferguson CJ, Chow CY, Jin N, Jones JM, Grant AE, et al. Pathogenic mechanism of the FIG4 mutation responsible for charcotmarie-tooth disease CMT4J. PLoS Genet. 2011;7 [cited 2020 Aug 10]. Available from: https://pubmed.ncbi.nlm.nih.gov/21655088/.

124. Renaud L, Picher-Martel V, Codron P, Julien JP. Key role of UBQLN2 in pathogenesis of amyotrophic lateral sclerosis and frontotemporal dementia. Acta Neuropathol Commun. 2019:103 [cited 2020 Jun 18]. Available from: https://actaneurocomms.biomedcentral.com/artic les/10.1186/s40478-019-0758-7.

125. Rademakers R, Baker M, Nicholson AM, Rutherford NJ, Finch N, SotoOrtolaza A, et al. Mutations in the colony stimulating factor 1 receptor (CSF1R) gene cause hereditary diffuse leukoencephalopathy with spheroids. Nat Genet. 2012;44:200-5 [cited 2020 Aug 10]. Available from: https://pubmed.ncbi.nlm.nih.gov/22197934/.

126. Pimenova AA, Raj T, Goate AM. Untangling genetic risk for Alzheimer's disease. Biol Psychiatry. 2017:300-10[cited 2018 Mar 27]. Available from:. https://doi.org/10.1016/j.biopsych.2017.05.014.

127. Jiang T, Hou J-K, Gao Q, Yu J-T, Zhou J-S, Zhao H-D, et al. TREM2 p.H157Y variant and the risk of Alzheimer's disease: a meta-analysis involving 14,510 subjects. Curr Neurovasc Res. 2016;13:318-20 [cited 2020 Aug 10]. Available from: https://pubmed.ncbi.nlm.nih.gov/ 27501831/.

128. Jin SC, Carrasquillo MM, Benitez BA, Skorupa T, Carrell D, Patel D, et al. TREM2 is associated with increased risk for Alzheimer's disease in African Americans. Mol Neurodegener. 2015;10:19 [cited 2018 Mar 16]. Available from: http://www.molecularneurodegeneration.com/ content/10/1/19.

129. Guerreiro R, Wojtas A, Bras J, Carrasquillo M, Rogaeva E, Majou-

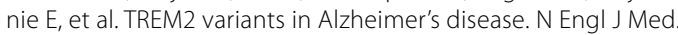
2013;368:117-27 [cited 2018 Mar 26]. Available from: http://www. nejm.org/doi/10.1056/NEJMoa1211851.

130. Rayaprolu S, Mullen B, Baker M, Lynch T, Finger E, Seeley WW, et al. TREM2 in neurodegeneration: evidence for association of the p.R47H variant with frontotemporal dementia and Parkinson's disease. Mol Neurodegener. 2013;8:19 [cited 2018 Mar 26]. Available from: http:// www.ncbi.nlm.nih.gov/pubmed/23800361.

131. Jiao B, Liu X, Tang B, Hou L, Zhou L, Zhang F, et al. Investigation of TREM2, PLD3, and UNC5C variants in patients with Alzheimer's disease from mainland China. Neurobiol Aging. 2014;35:2422.e9-2422. e11 [cited 2021 Jan 10]. Available from: https://pubmed.ncbi.nlm.nih. gov/24866402/.

132. Ma JF, Zhou Y, Xu J, Liu XH, Wang Y, Deng YL, et al. Association study of TREM2 polymorphism rs75932628 with late-onset Alzheimer's disease in Chinese Han population. Neurol Res. 2014;36:894-6 [cited 2021 Jan 10]. Available from: https://pubmed.ncbi.nlm.nih.gov/24725 293/.

133. Song W, Hooli B, Mullin K, Jin SC, Cella M, Ulland TK, et al. Alzheimer's disease-associated TREM2 variants exhibit either decreased or increased ligand-dependent activation. Alzheimers Dement. 2017;13:381-7 [cited 2021 Jan 10]. Available from: https://pubmed. ncbi.nlm.nih.gov/27520774/.

134. Sims R, Van Der Lee SJ, Naj AC, Bellenguez C, Badarinarayan N, Jakobsdottir J, et al. Rare coding variants in PLCG2, ABI3, and TREM2 implicate microglial-mediated innate immunity in Alzheimer's disease. Nat Genet. 2017:49:1373-84 [cited 2018 Mar 26]. Available from: https:// pubmed.ncbi.nlm.nih.gov/28714976/.

135. Albers PK, McVean G. Dating genomic variants and shared ancestry in population-scale sequencing data. PLoS Biol. 2020 Jan 17;18(1):e3000586. doi: 10.1371/journal.pbio.3000586.

136. Benton ML, Abraham A, LaBella AL, Abbot P, Rokas A, Capra JA. The influence of evolutionary history on human health and disease. Nat Rev Genet. 2021:269-83 [cited 2021 May 27]. Available from: www. nature.com/nrg.

137. McQuillan R, Leutenegger AL, Abdel-Rahman R, Franklin CS, Pericic M, Barac-Lauc L, et al. Runs of homozygosity in European populations. Am J Hum Genet. 2008;83:359-72 [cited 2020 Nov 12]. Available from: http://www.cell.com/article/S000292970800445X/fulltext.
138. Pemberton TJ, Absher D, Feldman MW, Myers RM, Rosenberg NA, Li JZ. Genomic patterns of homozygosity in worldwide human populations. Am J Hum Genet. 2012;91:275-92[cited 2020 Nov 12]. Available from:. https://doi.org/10.1016/j.ajhg.2012.06.014.

139. Ramachandran S, Deshpande O, Roseman CC, Rosenberg NA, Feldman MW, Cavalli-Sforza LL. Support from the relationship of genetic and geographic in human populations for a serial founder effect originating in Africa. Proc Natl Acad Sci U S A. 2005;102:15942-7 cited 2021 Apr 6]. Available from: http://www.pnas.org/cgi/doi/10.1073/ pnas.0507611102.

140. Coop G, Pickrell JK, Novembre J, Kudaravalli S, Li J, Absher D, et al. The role of geography in human adaptation. Schierup $\mathrm{MH}$, editor. PLoS Genet. 2009;5:e1000500[cited 2021 Apr 6]. Available from:. https:// doi.org/10.1371/journal.pgen.1000500.

141. Gravel S. When is selection effective? Genetics. 2016;203:451-62 [cited 2021 Jul 15]. Available from: /pmc/articles/PMC4858791/.

142. Zhao C, Strobino K, Moon YP, Cheung YK, Sacco RL, Stern Y, et al. APOE $\varepsilon 4$ modifies the relationship between infectious burden and poor cognition. Neurol Genet. 2020;6:e462 [cited 2021 Apr 13]. Available from: https://ng.neurology.org/content/6/4/e462.

143. Soscia SJ, Kirby JE, Washicosky KJ, Tucker SM, Ingelsson M, Hyman B, et al. The Alzheimer's disease-associated amyloid $\beta$-protein is an antimicrobial peptide. Bush Al, editor. PLoS One. 2010;5:e9505. https:// doi.org/10.1371/journal.pone.0009505.

144. Shen J, Kelleher RJ. The presenilin hypothesis of Alzheimer's disease: evidence for a loss-of-function pathogenic mechanism. Proc Natl Acad Sci. 2007; Available from: http://www.pnas.org/cgi/doi/10. 1073/pnas.0608332104.

145. Gosztyla ML, Brothers HM, Robinson SR. Alzheimer's amyloid- $\beta$ is an antimicrobial peptide: a review of the evidence. J Alzheimers Dis. 2018;62(4):1495-506. https://doi.org/10.3233/JAD-171133.

146. Cochran JN, McKinley EC, Cochran M, Amaral MD, Moyers BA, Lasseigne $\mathrm{BN}$, et al. Genome sequencing for early-onset or atypical dementia: high diagnostic yield and frequent observation of multiple contributory alleles. Cold Spring Harb Mol Case Stud. 2019:5:1-19.

147. Joutel A, Chabriat H, Vahedi K, Domenga V, Vayssiere C, Ruchoux MM, et al. Splice site mutation causing a seven amino acid Notch3 in-frame deletion in CADASIL. Neurology. 2000;54:1874-5 [cited 2018 Apr 3]. Available from: http://www.ncbi.nlm.nih.gov/pubmed/10802 807.

148. Arboleda-Velasquez JF, Lopera F, Lopez E, Frosch MP, SepulvedaFalla D, Gutierrez JE, et al. C455R notch3 mutation in a Colombian CADASIL kindred with early onset of stroke. Neurology. 2002;59:277-9 [cited 2018 Apr 3]. Available from: http://www.ncbi. nlm.nih.gov/pubmed/12136071.

149. Kremeyer B, Lopera F, Cox JJ, Momin A, Rugiero F, Marsh S, et al. A gain-of-function mutation in TRPA1 causes familial episodic pain syndrome. Neuron. 2010;66:671-80 [cited 2021 Jan 24]. Available from: https://pubmed.ncbi.nlm.nih.gov/20547126/.

150. Arango-Lasprilla JC, Iglesias-Dorado J, Moreno S, Lopera F. A neuropsychological study of Huntington's disease in families in Antioquia, Colombia. Rev Neurol. 2003;37:7-13 [cited 2021 Jun 21]. Available from: https://europepmc.org/article/med/12861500.

151. Cornejo-Olivas M, Torres L, Velit-Salazar MR, Inca-Martinez M, Mazzetti P, Cosentino C, et al. Variable frequency of LRRK2 variants in the Latin American research consortium on the genetics of Parkinson's disease (LARGE-PD), a case of ancestry. NPJ Parkinsons Dis. 2017;3:19. https:// doi.org/10.1038/s41531-017-0020-6.

152. Ramírez-Castro JL, Pineda-Trujillo N, Valencia AV, Muñetón CM, Botero O, Trujillo O, et al. Mutations in FOXL2 underlying BPES (types 1 and 2) in Colombian families. Am J Med Genet. 2002;113:47-51 [cited 2021 Jan 24]. Available from: https://pubmed.ncbi.nlm.nih.gov/12400 065/.

153. Pineda-Trujillo N, Carrizosa J, Cornejo W, Arias W, Franco C, Cabrera $D$, et al. A novel SCN1A mutation associated with severe GEFS+ in a large south American pedigree. Seizure. 2005;14:123-8.

154. Bouzaher $M H$, Worden $C P$, Jeyakumar A. Systematic review of pathogenic GJB2 variants in the Latino population. Otol Neurotol. 2020:e182-91 [cited 2020 Jun 17]. Available from: http://journals.lww. com/10.1097/MAO.0000000000002505. 
155. Torres D, Lorenzo Bermejo J, Rashid MU, Bricenõ I, Gil F, Beltran A, et al. Prevalence and penetrance of BRCA1 and BRCA2 germline mutations in Colombian breast cancer patients. Sci Rep. 2017;7:1-9.

156. Arboleda-Velasquez JF, Lopera F, O'Hare M, Delgado-Tirado S, Marino C, Chmielewska N, et al. Resistance to autosomal dominant Alzheimer's disease in an APOE3 Christchurch homozygote: a case report. Nat Med. 2019;25:1680-3[cited 2020 Oct 7]. Available from. https:// doi.org/10.1038/s41591-019-0611-3.

157. Acosta-Uribe J, Aguillon D, Cochran JN, Kosik KS, Lopera F. The admixture and Neurogeneration genetic landscape (TANGL) dataset. Medellín: Institutional Repository of the Universidad de Antioquia; 2022. Available from: https://doi.org/10.5062/F4N58JNW

\section{Publisher's Note}

Springer Nature remains neutral with regard to jurisdictional claims in published maps and institutional affiliations.

- fast, convenient online submission

- thorough peer review by experienced researchers in your field

- rapid publication on acceptance

- support for research data, including large and complex data types

- gold Open Access which fosters wider collaboration and increased citations

- maximum visibility for your research: over $100 \mathrm{M}$ website views per year

At BMC, research is always in progress.

Learn more biomedcentral.com/submissions 